\title{
ON CONGRUENCE LATTICES OF $\mathfrak{m}$-COMPLETE LATTICES
}

\author{
G. GRÄTZER and H. LAKSER
}

(Received 12 February 1990)

Communicated by T. E. Hall

\begin{abstract}
The lattice of all complete congruence relations of a complete lattice is itself a complete lattice. In an earlier paper, we characterize this lattice as a complete lattice. Let $m$ be an uncountable regular cardinal. The lattice $L$ of all $m$-complete congruence relations of an m-complete lattice $K$ is an m-algebraic lattice; if $K$ is bounded, then the unit element of $L$ is m-compact. Our main result is the converse statement: For an m-algebraic lattice $L$ with an m-compact unit element, we construct a bounded m-complete lattice $K$ such that $L$ is isomorphic to the lattice of $m$-complete congruence relations of $K$. In addition, if $L$ has more than one element, then we show how to construct $K$ so that it will also have a prescribed automorphism group. On the way to the main result, we prove a technical theorem, the One Point Extension Theorem, which is also used to provide a new proof of the earlier result.
\end{abstract}

1991 Mathematics subject classification (Amer. Math. Soc.) 08 A 65; 08 A 30.

Keywords and phrases: Complete lattice, m-complete lattice, complete congruence, m-complete congruence, congruence lattice, complete congruence lattice, automorphism group.

\section{Introduction}

In our earlier paper [8], we prove that the lattice $L$ of all complete congruence relations of a complete lattice $K$ can be characterized as a complete lattice; see also Theorem 8 in Section 6 of this paper. The basic technique we use, though not formalized there, could be called the "one-point extension": we construct a (complete) lattice in [8], the direct product of two well-ordered bounded chains, that contains all the (complete) congruences we need; then we adjoin an element each to a family of intervals in an obvious way. The

This research was supported by the NSERC of Canada.

(c) 1992 Australian Mathematical Society 0263-6115/92 \$A2.00+0.00 
resulting lattice has the required (complete) congruence lattice.

The construction in [8] is easy to follow because the lattice we deal with (the direct product of two chains) is easy to visualize. So we dispensed with the detailed calculation verifying that certain binary relations are (complete) congruences.

In this paper, our goal is to extend the main results of [8] from complete lattices to bounded $m$-complete lattices. To accomplish this, we have to apply the one-point construction to lattices that are direct products of two lattices neither of which is a chain; such lattices are much more complex than direct products of two chains. Therefore, the construction is difficult to visualize, and it is hard to justify the statements about congruence extensions without detailed computation. See the discussion in Section 8 on this point.

In Section 3, we formalize this construction. The main technical result, the "One Point Extension Theorem," gives necessary and sufficient conditions for the extendability of a congruence relation. Theorem 3 does this under the condition that none of the selected intervals is prime, while Theorem 6 settles the case where all selected intervals are prime. In the former case, all extension are unique; in the latter, we characterize which extensions are unique. The mixed case (prime and nonprime intervals) easily follows.

There is a special case of the One Point Extension Theorem, the "Colored Product Extension Theorem" (Theorem 7); the lattice to be extended is a direct product of two lattices, and the family of intervals comes from "coloring" the components. In this case, the conditions for extendability are easier to apply.

We first use these results to give a formal proof of the main result of [8] (Theorem 8). The reader can safely skip this section, since Theorem 13 contains Theorem 8, and its proof does not utilize Theorem 8. However, the reader may find it somewhat difficult to apply the "Colored Product Extension Theorem" directly to Theorem 13 without reading the proof of Theorem 8 .

For an uncountable regular cardinal $m$, we proceed to the $m$-complete case, and characterize the lattice $L$ of all $m$-complete congruence relations of a bounded m-complete lattice $K$ as an m-algebraic lattice with an mcompact unit element. In addition, we show how to construct such a $K$ with a prescribed automorphism group (Theorem 13). (This result was announced in [7].) The lattice $K$ we construct has some very special properties, see the Addendum to Theorem 13 in Section 8.

\section{Notation}

$\mathfrak{C}_{n}$ denotes the n-element chain with elements $0,1, \ldots, n-1$.

If $\alpha$ is an ordinal, then by an increasing chain $\left(x_{\gamma} \mid \gamma<\alpha\right)$ we mean a 
chain with $x_{\beta} \leq x_{\gamma}$ whenever $\beta \leq \gamma<\alpha$.

Let $\alpha$ be an ordinal, and for each $\gamma<\alpha$, let $A_{\gamma}$ be a lattice. We denote by $\Sigma\left(A_{\gamma} \mid \gamma<\alpha\right)$ the ordinal sum of the $A_{\gamma}$; for two components $A$ and $B$, let $A+B$ denote the ordinal sum of $A$ and $B$ (we place $B$ on top of A).

For ordinals $\alpha, \beta$, the ordinal product $\alpha \times \beta$ is regarded as the set $\{\langle\gamma, \delta\rangle \mid \gamma<\alpha, \delta<\beta\}$ ordered by $\left\langle\gamma_{1}, \delta_{1}\right\rangle \leq\left\langle\gamma_{2}, \delta_{2}\right\rangle$ if and only if $\gamma_{1}<\gamma_{2}$ or $\gamma_{1}=\gamma_{2}$ and $\delta_{1} \leq \delta_{2}$.

For a lattice $A$, the congruence lattice of $A$ is denoted by Con $A$. For a complete lattice $A$, the complete congruence lattice of $A$ is denoted by Com $A$.

For an interval $I=[u, v]$ in the lattice $A$, we shall denote by $\Theta_{A}(I)$ or $\Theta_{A}(u, v)$ the congruence relation generated by the interval $I$. If $A$ is understood, we use the notation $\Theta(I)$ or $\Theta(u, v)$. Note that $u \equiv v(\Theta)$ is equivalent to $\Theta(I) \leq \theta$. If $P$ is a set of intervals, then $\Theta(P)$ is the join of the $\Theta(I)$, with $I \in P$. Note that $\Theta(P) \leq \Theta$ means that $\Theta$ collapses all the intervals in $P$.

Let $\mathfrak{m}$ be an infinite regular cardinal. A lattice $K$ is $\mathfrak{m}$-complete if $\vee X$ and $\Lambda X$ exists in $K$ whenever $X \subseteq L$ and $0<|X|<\mathfrak{m}$. A congruence relation $\boldsymbol{\theta}$ of an $\mathfrak{m}$-complete lattice $K$ is an $\mathfrak{m}$-complete congruence relation if the Substitution Property holds for fewer than $\mathfrak{m}$ elements, that is, if $x_{i} \equiv y_{i}(\boldsymbol{\theta})$, for $i \in I$, and $|I|<\mathfrak{m}$, then

$$
\bigvee\left(x_{i} \mid i \in I\right) \equiv \bigvee\left(y_{i} \mid i \in I\right) \quad(\Theta)
$$

and dually.

An element $a$ of a complete lattice $L$ is m-compact if $a \leq \bigvee X$ implies that $a \leq \bigvee X_{1}$ for some subset $X_{1}$ of $X$ with $\left|X_{1}\right|<\mathfrak{m}$. The complete lattice $L$ is $\mathfrak{m}$-algebraic if every element of $L$ is a join of $\mathfrak{m}$-compact elements. We denote by comp $p_{m} L$ the set of nonzero m-compact elements of $L$.

The lattice $\operatorname{Com}_{\mathfrak{m}} K$ of all $\mathfrak{m}$-complete congruence relations of an $\mathfrak{m}$ complete lattice $K$ is an m-algebraic lattice.

For a lattice $A$, let Ip $A$ denote the the set of prime intervals in $A$, that is, the set of all intervals $\mathfrak{p}=[u, v]$, where $u \prec v$. If there are many prime intervals in $A$, then Ip $A$ plays an important role. This is the case if $A$ is strongly atomic, that is, if every proper interval of $A$ contains an atom.

The prime interior $\mathrm{pi}_{\mathfrak{m}} \boldsymbol{\theta}$ of an m-complete congruence relation $\boldsymbol{\theta}$ on an m-complete lattice $A$ is the m-complete congruence generated by all prime intervals of $A$ that are collapsed by $\Theta$. For a complete lattice and for a complete congruence $\Theta$, we define, similarly, pi $\theta$. 
We refer the reader to [3] for the standard notation in lattice theory.

We shall use the convention that Condition (2) of Theorem 3 (or Lemma 3 ) is referred to as Condition (3.2).

\section{The one point extension theorem}

In this section, we present a general theorem that proves very useful in representing congruence lattices of lattices; for some further applications of this result, see the announcement: [11], and the papers [9] and [10] by G. Grätzer and $\mathrm{H}$. Lakser.

Let $L$ be a lattice and let $\Lambda$ be a set of nontrivial intervals in $L$. We define a lattice $L^{\prime}=L[\Lambda]$ by adjoining the family of new pairwise distinct elements $\left\{m_{l} \mid I \in \Lambda\right\}$ to $L$, and, for each $I=[u, v] \in \Lambda$, requiring that $u \prec m_{I} \prec v$.

We associate with $x \in L^{\prime}$ the elements $\underline{x}$ and $\bar{x}$ of $L:$ for $x \in L$, set $\underline{x}=\bar{x}=x$; for $I=[u, v] \in \Lambda$ and the new element $m_{I}$, set $\underline{m}_{I}=u$ and $\bar{m}_{I}=v$. We then, more formally, define the relation $\leq_{L[\Lambda]}$ on the set $L[\Lambda]$ as follows:

$$
x \leq_{L[\Lambda]} y \quad \text { if } \quad x=y \quad \text { or } \quad \bar{x} \leq_{L} \underline{y},
$$

where $\leq_{L}$ denotes the partial ordering in $L$. We shall write $\leq$ for both $\leq_{L}$ and $\leq_{L[\Lambda]}$.

We then have the following lemma, whose proof is straightforward, and is left to the reader.

LEMMA 1. $\langle L[\Lambda], \leq\rangle$ is a lattice extending $L$. If $X$ is a subset of $L[\Lambda]$, then $\bigvee X$ exists in $L[\Lambda]$ if and only if either there is an $x \in X$ with $x \geq y$ whenever $y \in X$, in which case $\bigvee X=x$, or there is no such $x$ and $\bigvee_{L}(\bar{x})$ $x \in X$ ) exists, in which case

$$
\bigvee X=\bigvee_{L}(\bar{x} \mid x \in X)
$$

and dually for $\wedge$.

By Lemma 1, if $L$ is m-complete, then so is $L[\Lambda]$.

We shall determine which congruence relations on $L$ extend to $L[\Lambda]$ and, if $L$ is m-complete, which m-complete congruence relations on $L$ extend to m-complete congruence relations on $L[\Lambda]$.

We need the following extension of [3, Lemma I.8]. 
LEMMA 2. Let the reflexive binary relation $\Phi$ on the lattice $L$ satisfy the following two conditions whenever $x, y, z \in L$ :

(1) $x<y<z, x \equiv y(\Phi)$, and $y \equiv z$ (Ф) imply that $x \equiv z(\Phi)$;

(2) $x<y$ and $x \equiv y(\Phi)$ imply that

$$
x \vee z \equiv y \vee z \quad(\Phi) \quad \text { and } \quad x \wedge z \equiv y \wedge z \quad \text { (Ф). }
$$

Then the relation $\boldsymbol{\theta}$ defined by setting

$$
x \equiv y \quad(\Theta) \quad \text { if } \quad x \wedge y \equiv x \vee y
$$

is a congruence relation on $L$.

If, furthermore, $m$ is an infinite regular cardinal, $L$ is $\mathfrak{m}$-complete, and $\Phi$ satisfies, in addition, the condition

(3) If $0<\alpha<\mathfrak{m},\left(x_{\gamma} \mid \gamma<\alpha\right)$ and $\left(y_{\gamma} \mid \gamma<\alpha\right)$ are increasing chains in $L$, and, for all $\gamma<\alpha$, we have $x_{\gamma} \leq y_{\gamma}$ and $x_{\gamma} \equiv y_{\gamma}(\Phi)$, then $\bigvee\left(x_{\gamma} \mid \gamma<\alpha\right) \equiv \bigvee\left(y_{\gamma} \mid \gamma<\alpha\right)(\Phi)$;

and its dual, then $\boldsymbol{\theta}$ is $\mathrm{m}$-complete.

Proof. By [3, Lemma I.8], Conditions (2.1) and (2.2) imply that $\Theta$ is a congruence.

Now let $L$ be m-complete and let Conditions (2.1), (2.2), (2.3), and the dual of Condition (2.3) hold. Let $0<\alpha<\mathfrak{m}$, and let $\left(a_{\gamma} \mid \gamma<\alpha\right)$ and $\left(b_{\gamma} \mid \gamma<\alpha\right)$ be families of elements of $L$ with $a_{\gamma} \equiv b_{\gamma}(\boldsymbol{\theta})$ whenever $\gamma<\alpha$. By duality, it suffices to show that

$$
\bigvee\left(a_{\gamma} \mid \gamma<\alpha\right) \equiv \bigvee\left(b_{\gamma} \mid \gamma<\alpha\right) \quad(\boldsymbol{\theta})
$$

Since $\boldsymbol{\theta}$ is a congruence relation, we can assume that $a_{\gamma} \leq b_{\gamma}$ whenever $\gamma<\alpha$.

We proceed by transfinite induction on $\alpha$. Set $x_{0}=a_{0}, y_{0}=b_{0}$, and, for each $\gamma$ with $0<\gamma \leq \alpha$, set

$$
x_{\gamma}=\bigvee\left(a_{\beta} \mid \beta<\gamma\right), \quad y_{\gamma}=\bigvee\left(b_{\beta} \mid \beta<\gamma\right) .
$$

Then, by our induction hypothesis, for each $\gamma<\alpha$, we have

$$
x_{\gamma} \equiv y_{\gamma} \quad(\theta) \text {. }
$$

If $\alpha$ is a successor ordinal, $\alpha=\alpha^{\prime}+1$, then

$$
x_{\alpha}=x_{\alpha^{\prime}} \vee a_{\alpha^{\prime}} \equiv y_{\alpha^{\prime}} \vee b_{\alpha^{\prime}}=y_{\alpha} \quad(\Theta) \text {, }
$$

since $\theta$ is a congruence relation.

If $\alpha$ is a limit ordinal, then Condition (2.3) implies that $x_{\alpha} \equiv y_{\alpha}(\boldsymbol{\theta})$, concluding the proof of the lemma. 


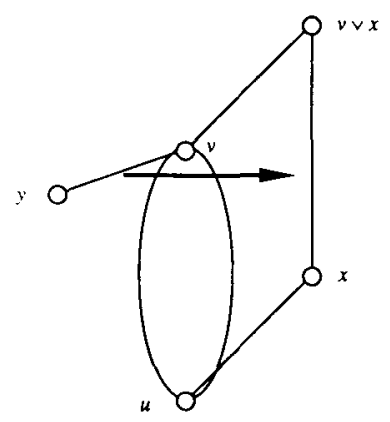

Condition (3.1)

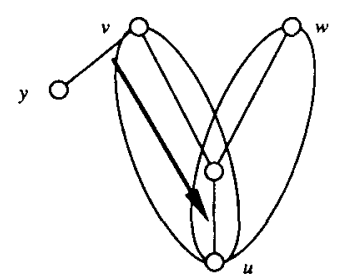

Condition (3.2)

FiguRE 1

In all of our applications, $\Lambda$ contains no prime interval. For the sake of simplicity, we shall make this assumption in the One Point Extension Theorem. For the general case, see Theorem 6 and the discussion following it. (The two conditions of the theorem are illustrated in Figure 1.)

Theorem 3 (One Point Extension Theorem). Let $\Lambda$ be a set of nontrivial, nonprime intervals in the lattice $L$, and let $\Theta$ be a congruence relation on $L$. Then $\Theta$ has an extension $\Theta[\Lambda]$ to $L[\Lambda]$ if and only if $\Theta$ satisfies the following conditions and their duals:

(1) for $[u, v] \in \Lambda, y<v$, and $u<x$,

$$
y \equiv v \quad(\boldsymbol{\Theta}) \quad \text { implies that } \quad v \vee x \equiv x \quad(\boldsymbol{\Theta}) \text {; }
$$

(2) for $[u, v],[u, w] \in \Lambda$, with $v \neq w$, and $y<v$,

$$
y \equiv v \quad(\boldsymbol{\Theta}) \quad \text { implies that } \quad v \wedge w \equiv u \quad(\boldsymbol{\Theta}) .
$$

The extension is unique.

If $L$ is an $\mathrm{m}$-complete lattice and $\Theta$ is an $\mathrm{m}$-complete congruence relation, then its extension $\Theta[\Lambda]$ is also m-complete.

Proof. Let us assume that the congruence relation $\theta$ of $L$ has an extension $\Theta^{\prime}$ to $L^{\prime}=L[\Lambda]$. Let $\Lambda, u, v, x, y$ be given as in Condition (3.1). Then $y \equiv v\left(\boldsymbol{\Theta}^{\prime}\right)$, and, taking the meet with $m_{I}$, we get $y \wedge u \equiv m_{I}\left(\boldsymbol{\Theta}^{\prime}\right)$, since $y \wedge m_{I}=y \wedge u$. Therefore, $m_{I} \equiv u\left(\boldsymbol{\Theta}^{\prime}\right)$. Taking the join with $x$, we get $v \vee x=m_{I} \vee x \equiv x(\boldsymbol{\Theta})$, since $\boldsymbol{\Theta}^{\prime}$ is an extension of $\boldsymbol{\Theta}$, establishing Condition (3.1).

Let $\Lambda, u, v, w, y$ be given as in Condition (3.2). As in the previous paragraph, $m_{[u, v]} \equiv u\left(\boldsymbol{\Theta}^{\prime}\right)$. Take any $z \in L[\Lambda]$ with $u<z<w$ and 
$z \neq m_{[u, v]}$. Then $z \vee m_{[u, v]} \equiv z \vee u\left(\boldsymbol{\Theta}^{\prime}\right)$, that is, $z \vee v \equiv z\left(\boldsymbol{\Theta}^{\prime}\right)$, and so meeting with $v \wedge w$ we get
(*)
$v \wedge w \equiv z \wedge v \wedge w$
$\left(\boldsymbol{\Theta}^{\prime}\right)$

Set $z=m_{[u, w]}$. Then we get

$$
v \wedge w \equiv m_{[u, w]} \wedge v \quad\left(\boldsymbol{\Theta}^{\prime}\right) .
$$

If $w \leq v$, then $m_{[u, w]} \wedge v=u$, and so

$$
v \wedge w \equiv u \quad(\boldsymbol{\theta})
$$

If $w \not \leq v$ fails, then $w<v$. Since the interval $[u, w]$ is not prime in $L$, there is an $x \in L$ with $u<x<w$. Setting $z=x$ in (*) yields the congruence $w \equiv x(\boldsymbol{\Theta})$, and so

$$
w \equiv m_{[u, w]} \wedge x \quad\left(\boldsymbol{\theta}^{\prime}\right) .
$$

Since $m_{[u, w]} \wedge x=u$, we again get

$$
v \wedge w=w \equiv u \quad(\boldsymbol{\theta}),
$$

thereby establishing Condition (3.2)

By duality, we establish the duals of Conditions (3.1) and (3.2).

Now let the congruence relation $\Theta$ on $L$ satisfy Conditions (3.1), (3.2), and their duals. We define a binary relation $\Phi$ on the pairs of comparable elements of $L^{\prime}=L[\Lambda]$.

For all $a \in L^{\prime}$, set $a \equiv a(\Phi)$. For all $a, b \in L^{\prime}$, with $a<b$, set

$$
a \equiv b
$$

if and only if the following three conditions hold:

\section{$\left(M_{a, b}\right) \quad \bar{a} \equiv \underline{b}(\boldsymbol{\theta})$;}

$\left(L_{a}\right) a \in L$ or $a \notin L$ and there is an

$$
x_{a} \in L \quad \text { with } x_{a}>\underline{a} \text { and } x_{a} \equiv \underline{a} \quad(\Theta) ;
$$

$\left(U_{b}\right) \quad b \in L$ or $b \notin L$ and there is a

$$
y_{b} \in L \quad \text { with } y_{b}<\bar{b} \text { and } y_{b} \equiv \bar{b} \quad(\boldsymbol{\Theta}) \text {. }
$$

We now prove that Conditions (2.1) and (2.2) hold for $\Phi$. Let $a, b$, $c \in L^{\prime}$, and let

$$
a<b<c, \quad a \equiv b \quad(\Phi), \quad \text { and } \quad b \equiv c \quad(\Phi) .
$$

Then Conditions $\left(L_{a}\right)$ and $\left(U_{c}\right)$ hold, and

$$
\bar{a} \equiv \underline{b} \quad(\Theta) \quad \text { and } \quad \bar{b} \equiv \underline{c} \quad(\Theta) .
$$


To prove that $a \equiv c(\Phi)$, we need only verify Condition $\left(M_{a, c}\right)$, which is equivalent to $\underline{b} \equiv \bar{b}(\boldsymbol{\theta})$. If $b \in L$, then this is trivial. On the other hand, if $b \notin L$, then by Conditions $\left(U_{b}\right)$ and $\left(L_{b}\right)$, there are $x_{b}, y_{b} \in L$ with

$$
x_{b}>\underline{b}, \quad x_{b} \equiv \underline{b} \quad(\boldsymbol{\theta}), \quad y_{b}<\bar{b}, \quad \text { and } \quad y_{b} \equiv \bar{b} \quad(\boldsymbol{\theta}) .
$$

Applying Condition (3.1) to $[\underline{b}, \bar{b}] \in \Lambda, u=\underline{b}, v=\bar{b}, y=y_{b}$, and $x=x_{b}$, we conclude that $x_{b} \equiv \bar{b}(\boldsymbol{\theta})$. Thus $\underline{b}=x_{b} \wedge y_{b} \equiv \bar{b} \wedge \bar{b}=\bar{b}(\boldsymbol{\theta})$, establishing Condition (2.1) for $\Phi$.

Now let $a, b, c \in L^{\prime}$ with $a<b$ and $a \equiv b(\Phi)$. To establish that $a \vee c \equiv b \vee c(\Phi)$, we may assume that $a<c$ and $b \not c$.

If $b>c$, then $a \vee c=c, b \vee c=b$ and

$$
\bar{a} \leq \underline{c} \leq \bar{c} \leq \underline{b} .
$$

Since $\bar{a} \equiv \underline{b}(\boldsymbol{\theta})$, we get that

$$
\underline{c} \equiv \bar{c} \quad(\boldsymbol{\theta}) \quad \text { and } \quad \bar{c} \equiv \underline{b} \quad(\boldsymbol{\theta}) .
$$

Thus Condition $\left(M_{c, b}\right)$ holds and, since $a \equiv b(\Phi)$, so does Condition $\left(U_{b}\right)$. To establish Condition $\left(L_{c}\right)$, we need only observe that if $c \notin L$, then $\bar{c}>\underline{c}$, and so we can set $x_{c}=\bar{c}$. Thus $c \equiv b(\Phi)$, that is, $a \vee c \equiv b \vee c$ $(\Phi)$ in case $b>c$.

We are left with the case: $a<c$ and $b, c$ are incomparable. Then

$$
\bar{a} \leq \underline{c}, \quad a \vee c=c, \quad \text { and } \quad b \vee c=\bar{b} \vee \bar{c} \in L,
$$

the last one from Lemma 1 . Joining $\bar{a} \equiv \underline{b}(\boldsymbol{\theta})$ with $\bar{c}$, we obtain that

$$
\bar{c} \equiv \underline{b} \vee \bar{c} \quad(\boldsymbol{\theta}) .
$$

If $b \in L$, then $\underline{b} \vee \bar{c}=\bar{b} \vee \bar{c}$; thus

$$
\underline{b} \vee \bar{c} \equiv \bar{b} \vee \bar{c}=b \vee c \quad(\boldsymbol{\theta}) .
$$

If $b \notin L$, then we get the $y_{b}$ from Condition $\left(U_{b}\right)$. Note that $\underline{b} \vee \bar{c}>\underline{b}$ follows from $c \not \leq b$. Then, applying Condition (3.1) to $[\underline{b}, \bar{b}] \in \Lambda, y=y_{b}$, and $x=\underline{b} \vee \bar{c}$, we conclude that $\underline{b} \vee \bar{c} \equiv \underline{b} \vee \bar{c} \vee \bar{b}(\theta)$, verifying ( $\ddagger$ ). Thus, in either event, we have ( $\dagger)$ and $(\ddagger)$, and so

$$
\overline{a \vee c}=\bar{c} \equiv b \vee c=\underline{b \vee c} \quad(\boldsymbol{\theta}),
$$

establishing Condition $\left(M_{a \vee c, b \vee c}\right)$.

$\left(U_{b \vee c}\right)$ holds since $b \vee c \in L$. If $c \in L$, then $\left(L_{a \vee c}\right)$ follows from $a \vee c=c$, and we are done. Assume, henceforth, that $c \notin L$. To conclude that

$$
c=a \vee c \equiv b \vee c \quad(\Phi),
$$


we need only establish Condition $\left(L_{c}\right)$, that is, we have to find an $x_{c} \in L$ with $x_{c}>\underline{c}$ and $x_{c} \equiv \underline{c}(\boldsymbol{\theta})$.

Since $\underline{b} \equiv \bar{a}(\boldsymbol{\theta})$, we get that

$$
\underline{b} \vee \underline{c} \equiv \bar{a} \vee \underline{c}=\underline{c} \quad(\boldsymbol{\theta}) .
$$

Thus if $\underline{c}<\underline{b} \vee \underline{c}$, that is, if $\underline{b} \underline{\underline{c}} \underline{c}$, then set $x_{c}=\underline{b} \vee \underline{c}>\underline{c}$.

If $\underline{b} \leq \underline{c}$, then $b \notin L$ follows from $b \not c$. By $\left(U_{b}\right)$, we then have $y_{b} \in L$ with $y_{b}<\bar{b}$ and $y_{b} \equiv \bar{b}(\boldsymbol{\theta})$.

Now if $\underline{b}<\underline{c}$, then, by Condition (3.1) applied to the interval $[\underline{b}, \bar{b}]$, $y_{b}$, and $x=\underline{c}$, we obtain that

$$
\bar{b} \vee \underline{c} \equiv \underline{c} \quad(\boldsymbol{\Theta}),
$$

and $\bar{b} \vee \underline{c}>\underline{c}$. In this case, set $x_{c}=\bar{b} \vee \underline{c}$.

If $\underline{b}=\underline{c}$, then applying Condition (3.2) to $[\underline{b}, \bar{b}],[\underline{c}, \bar{c}] \in \Lambda$ and $y_{b}<\bar{b}$ (recall that $y_{b} \equiv \bar{b}(\boldsymbol{\Theta})$ by Condition $\left(U_{b}\right)$ ), we get that

$$
\bar{b} \wedge \bar{c} \equiv \underline{c} \quad(\boldsymbol{\Theta}) .
$$

If $\bar{b} \wedge \bar{c}>\underline{c}$, then set $x_{c}=\bar{b} \wedge \bar{c}$. If $\bar{b} \wedge \bar{c}=\underline{c}$, then taking the meet of $(\ddagger)$ with $\bar{b}$, we conclude that

$$
\bar{b} \equiv \underline{c} \quad(\boldsymbol{\Theta}) .
$$

Since $\bar{b}>\underline{b}=\underline{c}$, we can set $x_{c}=\bar{b}$.

Therefore, if $a<c$ and $b, c$ are incomparable, then we conclude that $a \vee c \equiv b \vee c(\Phi)$, verifying Condition (2.2). By duality, the dual of Condition (2.2) holds.

Consequently, setting

$$
x \equiv y \quad(\Theta[\Lambda]) \quad \text { if } \quad x \wedge y \equiv x \vee y
$$

yields a congruence relation $\Theta[\Lambda]$ on $L[\Lambda]$ extending $\Theta$.

We now show that the extension of $\Theta$ to $L^{\prime}=L[\Lambda]$ is unique. Let the congruence relation $\boldsymbol{\Theta}^{\prime}$ on $L^{\prime}$ be an extension of $\boldsymbol{\theta}$. It suffices to show that if $a, b \in L^{\prime}$ with $a<b$ and $a \equiv b\left(\boldsymbol{\Theta}^{\prime}\right)$, then $a \equiv b(\Phi)$, that is, that Conditions $\left(M_{a, b}\right),\left(L_{a}\right)$, and $\left(U_{b}\right)$ hold.

Since

$$
a \leq \bar{a} \leq \underline{b} \leq b
$$

and $a \equiv b\left(\boldsymbol{\theta}^{\prime}\right)$, we conclude that

$$
a \equiv \bar{a} \quad\left(\boldsymbol{\Theta}^{\prime}\right), \quad \underline{b} \equiv b \quad\left(\boldsymbol{\Theta}^{\prime}\right), \quad \text { and } \quad \bar{a} \equiv \underline{b} \quad\left(\boldsymbol{\Theta}^{\prime}\right) .
$$

Thus $\bar{a} \equiv \underline{b}(\boldsymbol{\Theta})$, establishing Condition $\left(M_{a, b}\right)$.

To verify Condition $\left(U_{b}\right)$, we need only take the case $b \notin L$. Since the interval $[\underline{b}, \bar{b}]$ is not prime in $L$, there is a $y \in L$ with $\underline{b}<y<\bar{b}$. But 
$y$ is a relative complement of $b$ in the interval $[\underline{b}, \bar{b}]$ in $L^{\prime}$. Thus $\underline{b} \equiv b$ $\left(\boldsymbol{\Theta}^{\prime}\right)$ implies that $y \equiv \bar{b}(\boldsymbol{\Theta})$. Hence Condition $\left(U_{b}\right)$ is established by setting $y_{b}=y$.

The dual argument establishes Condition $\left(L_{a}\right)$. Thus we conclude that $\Theta^{\prime}=\Theta[\Lambda]$, verifying the uniqueness of the extension $\Theta[\Lambda]$.

Finally, let $\mathfrak{m}>\aleph_{0}$, let $L$ be $\mathfrak{m}$-complete, and let $\Theta$ be an $\mathfrak{m}$-complete congruence relation on $L$ satisfying Conditions (3.1), (3.2), and their duals. We show that the extension $\theta[\Lambda]$ of $\Theta$ is also $m$-complete. Define $\Phi$ by conditions $\left(M_{a, b}\right),\left(L_{a}\right)$, and $\left(U_{b}\right)$. We need only establish Condition (2.3) and its dual for $\Phi$.

Let $0<\alpha<\mathfrak{m}$, and let $\left(x_{\gamma} \mid \gamma<\alpha\right)$ and $\left(y_{\gamma} \mid \gamma<\alpha\right)$ be increasing chains in $L^{\prime}$ with $x_{\gamma} \leq y_{\gamma}$ and $x_{\gamma} \equiv y_{\gamma}(\Phi)$ whenever $\gamma<\alpha$. We make two claims.

Claim 4. Let $C$ be a chain of cardinality less than $m$ in $L^{\prime}$ such that

$$
\underline{x} \equiv x
$$

whenever $x \in C$. Then

$$
\bigvee(\underline{x} \mid x \in C) \equiv \bigvee C
$$

Proof. If there is a $c \in C$ such that $c \geq x$ whenever $x \in C$, then

$$
\bigvee C=c \text {. }
$$

If $x \leq c$, then $\underline{x} \leq \underline{c}$. Thus

$$
\bigvee(\underline{x} \mid x \in C)=\underline{c} .
$$

Since $\underline{c} \equiv c(\Phi)$, we conclude that $\bigvee(\underline{x} \mid x \in C) \equiv \bigvee C(\Phi)$.

Otherwise, for each $x \in C$, there is a $y \in C$ with $x<y$; in particular, $\bar{x} \leq \underline{y}$ holds. Then

$$
\bigvee(\bar{x} \mid x \in C)=\bigvee(\underline{x} \mid x \in C)
$$

But $\bigvee C=\bigvee(\bar{x} \mid x \in C)$, and so

$$
\bigvee(\underline{x} \mid x \in C)=\bigvee C,
$$

concluding the proof of Claim 4 .

ClaIm 5. Let $C$ be a chain of cardinality less than $m$ in $L^{\prime}$ such that

$$
x \equiv \bar{x}
$$

whenever $x \in C$. Then

$$
\bigvee C \equiv \bigvee(\bar{x} \mid x \in C)
$$


Proof. If there is a $c \in C$ such that $c \geq x$ whenever $x \in C$, then

$$
\bigvee C \equiv \bigvee(\bar{x} \mid x \in C) \quad(\Phi)
$$

exactly as in the proof of Claim 4 . Otherwise,

$$
\bigvee C=\bigvee(\bar{x} \mid x \in C) \text {, }
$$

concluding the proof of Claim 5.

Now let $B_{0}=\left\{\gamma<\alpha \mid x_{\gamma}=y_{\gamma}\right\}$, and let $B_{1}=\left\{\gamma<\alpha \mid x_{\gamma}<y_{\gamma}\right\}$. Then

$$
\bigvee\left(x_{\gamma} \mid \gamma \in B_{0}\right)=\bigvee\left(y_{\gamma} \mid \gamma \in B_{0}\right) \text {. }
$$

For $\gamma \in B_{1}$, we have $x_{\gamma} \leq \bar{x}_{\gamma} \leq \underline{y}_{\gamma} \leq y_{\gamma}$, and so

$$
\begin{array}{ll}
x_{\gamma} \equiv \bar{x}_{\gamma} & (\Phi), \\
\underline{y}_{\gamma} \equiv y_{\gamma} & (\Phi),
\end{array}
$$

and

$$
\bar{x}_{\gamma} \equiv \underline{y}_{\gamma} \quad(\boldsymbol{\theta}) .
$$

By Claims 4 and 5 ,

$$
\bigvee\left(x_{\gamma} \mid \gamma \in B_{1}\right) \equiv \bigvee\left(\bar{x}_{\gamma} \mid \gamma \in B_{1}\right)
$$

and

$$
\bigvee\left(y_{\gamma} \mid \gamma \in B_{1}\right) \equiv \bigvee\left(\underline{y}_{,}, \gamma \in B_{1}\right)
$$

Since $\boldsymbol{\theta}$ is m-complete, we also have

$$
\bigvee\left(\bar{x}_{\gamma} \mid \gamma \in B_{1}\right) \equiv \bigvee\left(\underline{y}_{\gamma} \mid \gamma \in B_{1}\right)
$$

Thus

$$
\bigvee\left(x_{\gamma} \mid \gamma \in B_{1}\right) \equiv \bigvee\left(y_{\gamma} \mid \gamma \in B_{1}\right)
$$

and so

$$
\bigvee\left(x_{\gamma} \mid \gamma<\alpha\right) \equiv \bigvee\left(y_{\gamma} \mid \gamma<\alpha\right)
$$

establishing Condition (2.3) for $\Phi$.

The dual argument holds for infinite meets, concluding the proof that if $\Theta$ is $\mathrm{m}$-complete, then so is its extension $\Theta[\Lambda]$, thereby concluding the proof of Theorem 3.

\section{The one point extension theorem in the general case}

We next handle the situation where all the intervals in $\Lambda$ are prime. Again, we use the notation $\Theta[\Lambda]$ to describe the binary relation on $L[\Lambda]$ 
defined as follows:

$$
x \equiv y \quad(\Theta[\Lambda]) \quad \text { if } \quad x \wedge y \equiv x \vee y \quad(\Phi),
$$

where $\Phi$ is defined on the pairs of comparable elements of $L[\Lambda]$ by the conditions $\left(M_{a, b}\right),\left(L_{a}\right)$, and $\left(U_{b}\right)$ of Section 3 .

THEOREM 6. Let $\Lambda$ be a set of prime intervals in the lattice $L$, and let $\Theta$ be a congruence relation on $L$. Then $\Theta$ has an extension to $L[\Lambda]$ if and only if $\boldsymbol{\Theta}$ satisfies Condition (3.1) and its dual, in which case, $\Theta[\Lambda]$ is an extension. The extension of $\boldsymbol{\theta}$ to $L[\Lambda]$ is unique if and only if $\boldsymbol{\Theta}$ also satisfies the following condition:

(1) If $[u, v] \in \Lambda$ is such that $x \equiv v$ (Ө) implies that $x \geq v$, then there is a $y>u$ with $y \nsupseteq v$;

and its dual.

If $L$ is an $\mathrm{m}$-complete lattice and $\Theta$ is an $\mathrm{m}$-complete congruence relation, then the extension $\Theta[\Lambda]$ is also $\mathrm{m}$-complete.

Proof. In the verification of the One Point Extension Theorem, we use the hypotheses that no interval is prime in the following steps: in the proof of the necessity of Condition (3.2) and its dual, and in the proof of the uniqueness of the extension. If all the intervals in $\Lambda$ are prime, then Condition (3.2) holds trivially. Consequently, the relation $\theta[\Lambda]$ is an extension of $\Theta$ in the present case also, and if $L$ and $\Theta$ are m-complete, then so is $\theta[\Lambda]$.

We now proceed to prove that Condition (6.1) together with its dual is a necessary and sufficient condition for the extension $\Theta[\Lambda]$ to be unique. We first establish the sufficiency.

Let Condition (6.1) and its dual hold, and let $\boldsymbol{\theta}^{\prime}$ be a congruence relation on $L^{\prime}=L[\Lambda]$ extending $\boldsymbol{\theta}$. We have to show that if $a, b \in L^{\prime}$ with $a<b$ and $a \equiv b\left(\boldsymbol{\Theta}^{\prime}\right)$, then $a \equiv b(\Phi)$, that is, that the pair $a, b$ satisfies Conditions $\left(M_{a, b}\right),\left(L_{a}\right)$, and $\left(U_{b}\right)$.

Since

$$
a \leq \bar{a} \leq \underline{b} \leq b
$$

and $a \equiv b\left(\boldsymbol{\Theta}^{\prime}\right)$, we get that

$$
a \equiv \bar{a} \quad\left(\boldsymbol{\Theta}^{\prime}\right), \quad \underline{b} \equiv b \quad\left(\boldsymbol{\Theta}^{\prime}\right), \quad \text { and } \quad \bar{a} \equiv \underline{b} \quad\left(\boldsymbol{\Theta}^{\prime}\right) .
$$

Thus $\bar{a} \equiv \underline{b}(\boldsymbol{\theta})$, establishing Condition $\left(M_{a, b}\right)$ for the pair $a, b$.

To establish Condition $\left(U_{b}\right)$, assume to the contrary that it fails: $b \notin L$ and there is no $y<\bar{b}$ in $L$ with $y \equiv \bar{b}(\boldsymbol{\theta})$. Then the hypothesis of Condition (6.1) holds for the interval $[\underline{b}, \bar{b}] \in \Lambda$. Consequently, there is a 
$y \in L$ with $y>\underline{b}$ and $y \nsucceq \bar{b}$. Since $\underline{b} \equiv b\left(\boldsymbol{\Theta}^{\prime}\right)$, taking the join with $y$, we get

$$
y=y \vee \underline{b} \equiv y \vee b=y \vee \bar{b} \quad\left(\Theta^{\prime}\right) .
$$

Taking the meet with $\bar{b}$ yields

$$
y \wedge \bar{b} \equiv \bar{b} \quad(\boldsymbol{\theta}),
$$

since both sides are elements of $L$. Since $y \nsupseteq \bar{b}$, we conclude that $y \wedge \bar{b}<\bar{b}$. Thus $y_{b}=y \wedge \bar{b}$ satisfies $\left(U_{b}\right)$, a contradiction. Thus Condition $\left(U_{b}\right)$ is verified.

The dual argument establishes Condition $\left(L_{a}\right)$.

Therefore, $a \equiv b(\Phi)$, proving the sufficiency of Condition (6.1) and its dual for the uniqueness of the extension of $\boldsymbol{\theta}$.

To prove the necessity of Condition (6.1) and its dual, we assume that either Condition (6.1) or its dual fails, and describe two distinct extensions of $\boldsymbol{\theta}$. By duality, we may assume that Condition (6.1) fails: there is an interval $I=[u, v] \in \Lambda$ satisfying, for all $x \in L$,

(*) $x \equiv v(\theta)$ implies that $x \geq v$;

(**) $x>u$ implies that $x \geq v$.

By Conditions $(*)$ and $\left(U_{b}\right)$,

$$
u \not \equiv m_{I} \quad(\Theta[\Lambda]) \text {. }
$$

Define the equivalence relation $\Theta_{1}$ on $L^{\prime}$ by setting its only nontrivial equivalence class to be $\left\{u, m_{I}\right\}$. We claim that $\boldsymbol{\theta}_{1}$ is a congruence relation on $L^{\prime}$.

To show that $\boldsymbol{\theta}_{1}$ preserves $\vee$, we take $c \in L^{\prime}$ with $c>u, c \neq m_{I}$, and show that $c=c \vee u \equiv c \vee m_{I}\left(\boldsymbol{\Theta}_{1}\right)$. If $\underline{c}>u$, then $c \geq \underline{c} \geq v$ by Condition (**), and so $c=c \vee m_{I}$. If $\underline{c}=u$, then $c \notin L$, and so $\bar{c}>u>\underline{c}$ by Condition (**). This contradicts the hypothesis that all members of $\Lambda$ are prime. Consequently, $\theta_{1}$ preserves $\vee$.

As for $\wedge$, we need only consider $c<m_{I}$. Then $c \leq u$, and so $c \wedge m_{I}=$ $c \wedge u$.

Therefore, $\boldsymbol{\theta}_{1}$ is a congruence relation on $L^{\prime}$, which clearly is an extension of the trivial congruence relation $\omega_{L}$ on $L$. The congruence relation $\Theta[\Lambda] \vee \Theta_{1}$ is distinct from $\Theta[\Lambda]$ since $u \not \equiv m_{I}(\Theta[\Lambda])$. It is easy to see that

$$
\Theta[\Lambda] \vee \Theta_{1}=\left(\Theta[\Lambda] \circ \Theta_{1}\right) \cup\left(\Theta_{1} \circ \Theta[\Lambda]\right),
$$

where $\circ$ denotes the relation theoretic product. Thus $\theta[\Lambda] \vee \Theta_{1}$ is also an extension of $\boldsymbol{\theta}$. It is also easy to see that if $L$ and $\theta$ are m-complete, then so is $\theta[\Lambda] \vee \theta_{1}$. Thus the nonuniqueness of the extension is established, showing the equivalence of the uniqueness of the extension with Condition (6.1) and its dual. This completes the proof of Theorem 6. 
In the case of an arbitrary $\Lambda$, set $\Lambda_{1}$ equal to the set of prime intervals in $\Lambda$, and set $\Lambda_{0}$ equal to the set of nonprime intervals in $\Lambda$. Then

$$
L[\Lambda] \cong L\left[\Lambda_{0}\right]\left[\Lambda_{1}\right] .
$$

A congruence relation $\Theta$ on $L$ extends to $L[\Lambda]$ if and only if $\Theta$ extends to $\Theta\left[\Lambda_{0}\right]$ on $L\left[\Lambda_{0}\right]$, where the One Point Extension Theorem applies, and $\Theta\left[\Lambda_{0}\right]$ extends to $L\left[\Lambda_{0}\right]\left[\Lambda_{1}\right]$, where Theorem 6 applies. Also, the extension of $\Theta$ to $L[\Lambda]$ is unique if and only if the extension of $\Theta\left[\Lambda_{0}\right]$ to $L[\Lambda]$ is unique.

\section{Products of colored lattices}

In [8], we generalized the concept of a coloring of a lattice $L$ introduced by S.-K. Teo [14] for chains, whereby the prime intervals of $L$ are labelled by the elements of some set. We further generalize this concept here by not requiring that the intervals be prime nor that all prime intervals be labelled.

A (generalized) coloring $\varphi$ of a lattice $L$ by a set $X$ is a surjective (onto) mapping $\varphi: P \rightarrow X$, where $P$ is a set of nontrivial intervals in $L$.

Let $C$ be a chain with a unit 1 , and let $\varphi: P \rightarrow X$ be a coloring of $C$, where $P$ is a set of nontrivial intervals of $C$. We call $\varphi$ repetitious if Ip $C \subseteq P$ and

$$
(\operatorname{Ip}[u, 1]) \varphi=X
$$

whenever $u \in C$ with $u<1$. The coloring in Section 6 and the first coloring in Section 7 are repetitious.

For $i=0,1$, let $A_{i}$ be a lattice with a coloring $\varphi_{i}: P_{i} \rightarrow X$. We define a set $\Lambda$ of intervals in $A_{0} \times A_{1}$ by setting

$$
\Lambda=\left\{I_{0} \times I_{1} \mid I_{0} \in P_{0}, I_{1} \in P_{1}, \text { and } I_{0} \varphi_{0}=I_{1} \varphi_{1}\right\}
$$

Let us denote the lattice $\left(A_{0} \times A_{1}\right)[\Lambda]$ by $A_{0} \times{ }_{\varphi} A_{1}$, and the element $m_{I_{0} \times I_{1}}$ by $m\left(I_{0}, I_{1}\right)$.

In this section, we use the One Point Extension Theorem to determine the congruence relations on $A_{0} \times A_{1}$ that extend to $A_{0} \times{ }_{\varphi} A_{1}$. Recall that any congruence relation $\Theta$ on the lattice $A=A_{0} \times A_{1}$ is of the form $\Theta_{0} \times \Theta_{1}$, where, for $i=0,1, \Theta_{i}$ is a congruence relation on $A_{i}$; and that $A$ is m-complete if and only if $A_{0}$ and $A_{1}$ are m-complete, and, in that event, that $\boldsymbol{\Theta}$ is $m$-complete if and only if $\Theta_{0}$ and $\Theta_{1}$ are m-complete.

Theorem 7 (Colored Product Extension Theorem). The congruence relation $\boldsymbol{\Theta}=\Theta_{0} \times \Theta_{1}$ on $A_{0} \times A_{1}$ extends to $A_{0} \times{ }_{\varphi} A_{1}$ if and only if the following 
two conditions and their duals hold:

(1) For $I_{0} \in P_{0}, I_{1} \in P_{1}$, if $I_{0} \varphi_{0}=I_{1} \varphi_{1}$, then

$$
\boldsymbol{\Theta}\left(I_{0}\right) \leq \boldsymbol{\Theta}_{0} \text { is equivalent to } \boldsymbol{\Theta}\left(I_{1}\right) \leq \boldsymbol{\theta}_{1} ;
$$

(2) For $i=0,1$, if $I=[u, v] \in P_{i}$ and there is $a y<v$ with $y \equiv v$ $\left(\boldsymbol{\theta}_{i}\right)$, then $\boldsymbol{\theta}(I) \leq \boldsymbol{\theta}_{i}$.

In that event, the extension is unique.

If, in addition, $A_{0}$ and $A_{1}$ are $\mathfrak{m}$-complete and $\boldsymbol{\Theta}$ is $\mathfrak{m}$-complete, then so is its extension to $A_{0} \times{ }_{\varphi} A_{1}$.

Proof. Since none of the intervals in $\Lambda$ are prime, the One Point Extension Theorem applies. We note that in the present case Condition (3.1) is equivalent to the stronger condition:

$\left(3.1^{+}\right)$If $[u, v] \in \Lambda$ and there is a $y<v$ with $y \equiv v(\boldsymbol{\theta})$, then $u \equiv v$ $(\theta)$.

Indeed, if $u=\left\langle u_{0}, u_{1}\right\rangle, v=\left\langle v_{0}, v_{1}\right\rangle, x=\left\langle u_{0}, v_{1}\right\rangle$ then, by Condition (3.1),

$$
\left\langle v_{0}, v_{1}\right\rangle=\left\langle u_{0}, v_{1}\right\rangle \vee\left\langle v_{0}, v_{1}\right\rangle \equiv\left\langle u_{0}, v_{1}\right\rangle \quad(\Theta),
$$

and, similarly,

$$
\left\langle v_{0}, v_{1}\right\rangle \equiv\left\langle v_{0}, u_{1}\right\rangle \quad(\boldsymbol{\theta})
$$

Consequently,

$$
\left\langle v_{0}, v_{1}\right\rangle \equiv\left\langle u_{0}, v_{1}\right\rangle \wedge\left\langle v_{0}, u_{1}\right\rangle=\left\langle u_{0}, u_{1}\right\rangle \quad(\Theta),
$$

establishing Condition $\left(3.1^{+}\right)$.

Note also that Condition (3.2) follows immediately from Condition $\left(3.1^{+}\right)$. Thus, in view of the One Point Extension Theorem, we need only show that the conjunction of Conditions (7.1) and (7.2) is equivalent to Condition $\left(3.1^{+}\right)$.

Let Conditions (7.1) and (7.2) hold. Let $\left[\left\langle u_{0}, u_{1}\right\rangle,\left\langle v_{0}, v_{1}\right\rangle\right] \in \Lambda$, and let $\left\langle y_{0}, y_{1}\right\rangle\left\langle\left\langle v_{0}, v_{1}\right\rangle\right.$ with $\left\langle y_{0}, y_{1}\right\rangle \equiv\left\langle v_{0}, v_{1}\right\rangle(\boldsymbol{\theta})$. Without loss of generality, we may assume that $y_{0}<v_{0}$. Since $y_{0} \equiv v_{0}\left(\Theta_{0}\right)$, it follows from Condition (7.2) that $u_{0} \equiv v_{0}\left(\Theta_{0}\right)$. Since $\left[u_{0}, v_{0}\right] \varphi_{0}=\left[u_{1}, v_{1}\right] \varphi_{1}$, it follows from Condition (7.1) that $u_{1} \equiv v_{1}\left(\boldsymbol{\Theta}_{1}\right)$, which, by symmetry, establishes Condition (3. $\left.1^{+}\right)$.

Now let Condition $\left(3.1^{+}\right)$hold. Let $\left[u_{0}, v_{0}\right] \varphi_{0}=\left[u_{1}, v_{1}\right] \varphi_{1}$, and let $u_{0} \equiv v_{0}\left(\boldsymbol{\Theta}_{0}\right)$. Then $\left[\left\langle u_{0}, u_{1}\right\rangle,\left\langle v_{0}, v_{1}\right\rangle\right] \in \Lambda,\left\langle u_{0}, v_{1}\right\rangle<\left\langle v_{0}, v_{1}\right\rangle$, and $\left\langle u_{0}, v_{1}\right\rangle \equiv\left\langle v_{0}, v_{1}\right\rangle(\Theta)$. Thus by Condition $\left(3.1^{+}\right),\left\langle u_{0}, u_{1}\right\rangle \equiv\left\langle v_{0}, v_{1}\right\rangle$ $(\Theta)$, and so $u_{1} \equiv v_{1}\left(\Theta_{1}\right)$; by symmetry, this establishes Condition (7.1).

Let $i=0,\left[u_{0}, v_{0}\right] \in P_{0}, y<v_{0}$, and $y \equiv v_{0}\left(\Theta_{0}\right)$. Since $\varphi_{1}$ is surjective, there is an interval $\left[u_{1}, v_{1}\right] \in P_{1}$ with $\left[u_{0}, v_{0}\right] \varphi_{0}=\left[u_{1}, v_{1}\right] \varphi_{1}$, 
that is, with $\left[\left\langle u_{0}, u_{1}\right\rangle,\left\langle v_{0}, v_{1}\right\rangle\right] \in \Lambda$. Now $\left\langle y, v_{1}\right\rangle<\left\langle v_{0}, v_{1}\right\rangle$ and $\left\langle y, v_{1}\right\rangle \equiv$ $\left\langle v_{0}, v_{1}\right\rangle(\boldsymbol{\Theta})$. Then, by Condition $\left(3.1^{+}\right),\left\langle u_{0}, u_{1}\right\rangle \equiv\left\langle v_{0}, v_{1}\right\rangle(\boldsymbol{\Theta})$, and so $u_{0} \equiv v_{0}\left(\Theta_{0}\right)$, establishing Condition (7.2) for $i=0$. By symmetry, we get the proof for the case $i=1$.

Thus the conjunction of Conditions (7.1) and (7.2) is equivalent to Condition $\left(3.1^{+}\right)$. Theorem 7 then follows by the One Point Extension Theorem.

\section{An application to complete lattices}

In [8], we proved the following result:

THEOREM 8. Every complete lattice $L$ is isomorphic to the complete congruence lattice of a suitable complete lattice $K$.

In this section, we recall the construction of $K$, and show how the One Point Extension Theorem and the Colored Product Extension Theorem can be applied to prove this result.

In [8], we construct a well-ordered bounded chain $C$, with smallest element $0^{C}$ and largest element $1^{C}$, and a coloring

$$
\varphi: \text { Ip } C \rightarrow L-\{0\}
$$

with $\left[0^{C}, a^{C}\right] \varphi=1$, where $a^{C}$ is the cover of $0^{C}$. Furthermore, for each nonempty subset $X$ of $L-\{0\}$, there is an interval $X^{\dagger}=\left[0^{X}, 1^{X}\right]$ in $C$ such that $\left[0^{X}, j^{X}\right] \varphi=\bigvee_{L} X$, where $j^{X}$ is the cover of $0^{X}$, and, such that the coloring $\varphi$ restricted to $\left[j^{X}, 1^{X}\right]$ is repetitious, that is, $\left(\operatorname{Ip}\left[u, 1^{X}\right]\right) \varphi=X$ whenever $0^{X}<u<1^{X}$. In addition, $1^{X}$ has no lower cover. Finally, if $X \neq Y$, then $X^{\dagger} \cap Y^{\dagger}$ contains at most one element. (In [8], we actually have $X^{\dagger} \cap Y^{\dagger}=\varnothing$.) We then set

$$
C(\varphi)=C \times{ }_{\varphi} C,
$$

and define the set of intervals $\Lambda$ in $C(\varphi)$ by

$$
\Lambda=\left\{X^{\dagger} \times\left\{0^{C}\right\} \mid \varnothing \neq X \subseteq L-\{0\}\right\} .
$$

We then define the complete lattice $K$ as follows:

$$
K=C(\varphi)[\Lambda]
$$

In [8], we proved the following result.

LemMA 9 ([8, Lemma 1]). In a strongly atomic complete lattice $A$, the equality

$$
\text { pi } \boldsymbol{\theta}=\boldsymbol{\Theta}
$$


holds for any complete congruence relation $\Theta$ of $A$.

To determine Com $K$, we prove the following two lemmas.

LEMMA 10. A complete congruence relation $\Phi$ on $C^{2}$ has an extension to $C(\varphi)$ if and only if $\Phi=\Theta^{2}$ where $\boldsymbol{\theta}$ is a complete congruence relation on $C$ satisfying the condition:

(1) If $\mathfrak{p}, \mathfrak{q}$ are prime intervals in $C$ with $\mathfrak{p} \varphi=\mathfrak{q} \varphi$, then

$$
\boldsymbol{\Theta}(\mathfrak{p}) \leq \boldsymbol{\theta} \text { is equivalent to } \boldsymbol{\Theta}(\mathfrak{q}) \leq \boldsymbol{\theta} .
$$

The extension is unique.

Proof. This lemma follows immediately from the Colored Product Extension Theorem. Since $P_{0}=P_{1}=\operatorname{Ip} C$ and $C$ is a chain, it follows that Condition (7.2) and its dual hold. Moreover, since any well-ordered chain is strongly atomic, we can apply Lemma 9 to prove that Condition (10.1) is equivalent, in this case, to Condition (7.1).

LEMMA 11. A complete congruence relation $\Phi$ on $C^{2}$ has an extension to $K=C(\varphi)[\Lambda]$ if and only if $\Phi=\Theta^{2}$, where $\boldsymbol{\theta}$ is a complete congruence relation on $C$ satisfying the condition:

(1) If $P$ is a set of prime intervals in $C$, $q$ is a prime interval in $C$ with $\mathfrak{q} \varphi \leq \bigvee_{L} P \varphi$, and $\boldsymbol{\Theta}(\boldsymbol{P}) \leq \boldsymbol{\Theta}$, then $\boldsymbol{\Theta}(\mathfrak{q}) \leq \boldsymbol{\Theta}$.

The extension is unique.

Proof. Let $\Phi=\Theta^{2}$ and $\Theta \in \operatorname{Com} C$; let us assume that Condition (11.1) holds for $\Theta$. Note that the special case $P=\{\mathfrak{p}\}$ of (11.1) implies that Condition (10.1) holds. Thus $\Phi$ extends uniquely to a complete congruence $\boldsymbol{\theta}(\varphi)$ on $C(\varphi)$. We now apply the One Point Extension Theorem to show that $\Theta(\varphi)$ extends uniquely to $K$.

Note that Condition (3.2) and its dual hold trivially. Thus we need only establish Condition (3.1) and its dual.

Let $\varnothing \neq X \subseteq L-\{0\}$. We have to show that the interval

$$
\left[\left\langle 0^{X}, 0^{C}\right\rangle,\left\langle 1^{X}, 0^{C}\right\rangle\right]
$$

of $C(\varphi)$ satisfies Condition (3.1) and its dual. Let $y<\left\langle 1^{X}, 0^{C}\right\rangle$ with $y \equiv$ $\left\langle 1^{X}, 0^{C}\right\rangle(\Theta(\varphi))$. We shall prove that this implies that

$$
\left\langle 0^{X}, 0^{C}\right\rangle \equiv\left\langle 1^{X}, 0^{C}\right\rangle \quad(\Theta(\varphi)),
$$

a statement stronger than Condition (3.1). 
Indeed, there is a $y_{0} \in C$, with $j^{X} \leq y_{0}<1^{X}$ and $y \leq\left\langle y_{0}, 0^{C}\right\rangle$. For each $x \in X$, there is a $\mathfrak{p} \in \operatorname{Ip}\left[y_{0}, 1^{X}\right]$ with $\mathfrak{p} \varphi=x$. Since $y_{0} \equiv 1^{X}(\boldsymbol{\theta})$, it follows that $\boldsymbol{\theta}(\mathfrak{p}) \leq \boldsymbol{\theta}$. Thus by $(10.1), \boldsymbol{\theta}(\mathfrak{q}) \leq \boldsymbol{\theta}$ for each prime interval $\mathfrak{q}$ of $C$ with $q \varphi \in X$. Since $\left(\operatorname{Ip}\left[j^{X}, 1^{X}\right]\right) \varphi=X$ and $C$ is strongly atomic, we conclude that

$$
j^{X} \equiv 1^{X}
$$

Since $\left[0^{X}, j^{X}\right]$ is a prime interval and

$$
\left[0^{X}, j^{X}\right] \varphi=\bigvee X=\bigvee\left(\operatorname{Ip}\left[j^{X}, 1^{X}\right]\right) \varphi,
$$

we conclude by Condition (11.1) that

$$
0^{X} \equiv j^{X} \quad(\boldsymbol{\theta}) \text {. }
$$

Thus $0^{X} \equiv 1^{X}(\Theta)$, and so $\left\langle 0^{X}, 0^{C}\right\rangle \equiv\left\langle 1^{X}, 0^{C}\right\rangle(\Theta(\varphi))$, as claimed.

To establish the dual of Condition (3.1) for the interval

$$
\left[\left\langle 0^{X}, 0^{C}\right\rangle,\left\langle 1^{X}, 0^{C}\right\rangle\right],
$$

we again prove a stronger statement: if $y>\left\langle 0^{X}, 0^{C}\right\rangle$ and $y \equiv\left\langle 0^{X}, 0^{C}\right\rangle$ $(\Theta(\varphi))$, then $\left\langle 0^{X}, 0^{C}\right\rangle \equiv\left\langle 1^{X}, 0^{C}\right\rangle(\Theta(\varphi))$. Note that if $\left.y\right\rangle\left\langle 0^{X}, 0^{C}\right\rangle$, then $y \geq\left\langle j^{X}, 0^{C}\right\rangle$, or $y \geq\left\langle 0^{X}, a^{c}\right\rangle$, or $\vee X=1$ and

$$
y \geq m\left(\left[0^{X}, j^{X}\right],\left[0^{C}, a^{C}\right]\right) .
$$

Therefore,

$$
y \equiv\left\langle 0^{X}, 0^{C}\right\rangle \quad(\boldsymbol{\Theta}(\varphi))
$$

implies that either $0^{X} \equiv j^{X}(\Theta)$ or $0^{C} \equiv a^{C}(\Theta)$.

Let $0^{C} \equiv a^{C}(\Theta)$. Since $\left[0^{C}, a^{C}\right] \varphi=1$, we conclude from Condition (10.1) that $\Theta(\mathfrak{p}) \leq \boldsymbol{\theta}$ whenever $\mathfrak{p} \in \mathrm{Ip} C$; just set $P=\left\{\left[0^{C}, a^{C}\right]\right\}$. Then certainly $\left\langle 0^{X}, 0^{C}\right\rangle \equiv\left\langle 1^{X}, 0^{C}\right\rangle(\Theta(\varphi))$, as claimed.

On the other hand, let $0^{X} \equiv j^{X}(\Theta)$. Then set $P=\left\{\left[0^{X}, j^{X}\right]\right\}$. For each $\mathfrak{p} \in \operatorname{Ip}\left[j^{X}, 1^{X}\right]$, we have

$$
\mathfrak{p} \varphi \leq \bigvee X=\left[0^{X}, j^{X}\right] \varphi,
$$

and so $\Theta(\mathfrak{p}) \leq \boldsymbol{\theta}$ by Condition (11.1). Since $C$ is strongly atomic, we conclude that $j^{X} \equiv 1^{X}(\Theta)$. Thus $0^{X} \equiv 1^{X}(\Theta)$, and so $\left\langle 0^{X}, 0^{C}\right\rangle \equiv\left\langle 1^{X}, 0^{C}\right\rangle$ $(\Theta(\varphi))$, as required.

Thus Condition (11.1) implies that $\Phi$ extends uniquely to $K$.

Now assume that $\Phi$ extends to $K$. Then $\Phi$ certainly extends to $C(\varphi)$, and thus $\Phi=\Theta^{2}$. We observe first that $\Theta$ satisfies Condition (10.1). Thus to prove that $\boldsymbol{\theta}(\mathfrak{p}) \leq \boldsymbol{\theta}$ for a prime interval $\mathfrak{p}$, it suffices to find a prime interval $\mathfrak{p}^{\prime}$ satisfying $\boldsymbol{\Theta}\left(\mathfrak{p}^{\prime}\right) \leq \boldsymbol{\theta}$ and $\mathfrak{p}^{\prime} \varphi=\mathfrak{p} \varphi$. 
We now establish Condition (11.1) for $\Theta$. Set $X=P \varphi, Y=X \cup\{q \varphi\}$. Since $\Theta(P) \leq \Theta$ whenever $p$ is a prime interval in $\left[j^{X}, 1^{X}\right]$, we see that $\boldsymbol{\Theta}(\mathfrak{p}) \leq \boldsymbol{\Theta}$. Thus by Lemma $9, j^{X} \equiv 1^{X}(\boldsymbol{\Theta})$ and so

$$
\left\langle j^{X}, 0^{C}\right\rangle \equiv\left\langle 1^{X}, 0^{C}\right\rangle \quad(\boldsymbol{\Theta}(\varphi)) .
$$

We apply Condition (3.1) to the interval $\left[\left\langle 0^{X}, 0^{C}\right\rangle,\left\langle 1^{X}, 0^{C}\right\rangle\right] \in \Lambda, y=$ $\left\langle j^{X}, 0^{C}\right\rangle, x=\left\langle 0^{X}, a^{C}\right\rangle$, to conclude that

$$
\left\langle 0^{X}, a^{C}\right\rangle \equiv\left\langle 1^{X}, a^{C}\right\rangle \quad(\Theta(\varphi)),
$$

and so $0^{X} \equiv j^{X}(\boldsymbol{\theta})$. Since

$$
\left[0^{X}, j^{X}\right] \varphi=\bigvee X=\bigvee Y=\left[0^{Y}, j^{Y}\right] \varphi,
$$

we conclude by $(10.1)$ that

$$
\left\langle 0^{Y}, 0^{C}\right\rangle \equiv\left\langle j^{Y}, 0^{C}\right\rangle \quad(\boldsymbol{\Theta}(\varphi)) .
$$

There is a prime interval $[a, b]$ in $\left[j^{Y}, 1^{Y}\right]$ with $[a, b] \varphi=q \varphi$. Note that $b<1^{Y}$, since $1^{Y}$ has no lower cover. By the dual of Condition (3.1), applied to the interval $\left[\left\langle 0^{Y}, 0^{C}\right\rangle,\left\langle 1^{Y}, 0^{C}\right\rangle\right] \in \Lambda, y=\left\langle j^{Y}, 0^{C}\right\rangle, x=\left\langle b, 0^{C}\right\rangle$, we conclude that

$$
\left\langle 0^{Y}, 0^{C}\right\rangle \equiv\left\langle b, 0^{C}\right\rangle \quad(\boldsymbol{\Theta}(\varphi)),
$$

and thus $a \equiv b(\boldsymbol{\Theta})$. Consequently, $\boldsymbol{\Theta}(\mathfrak{q}) \leq \boldsymbol{\Theta}$, thereby establishing Condition (11.1) for $\theta$.

The proof of Lemma 11 is thus concluded.

The following isomorphism completes the proof of Theorem 8 .

\section{Lemma 12 . $\operatorname{Com} K \cong L$.}

Proof. As in [8], for each $x \in L$, we define a binary relation $\Phi^{x}$ on $C$ as follows:

for $u, v \in C$ with $u \leq v$, we set

$$
u \equiv v \quad\left(\Phi^{x}\right) \quad \text { if } \quad \mathfrak{p} \varphi \leq x \text { for every } \mathfrak{p} \in \operatorname{Ip}[u, v] .
$$

Since $C$ is a complete chain, it is clear that $\Phi^{x}$ is a complete congruence relation on $C$, and equally clearly, $\Phi^{x}$ satisfies Condition (11.1). Thus the complete congruence relation $\left(\Phi^{x}\right)^{2}$ of $C^{2}$ extends uniquely to a complete congruence relation $\Theta^{x}$ of $K$. Define

$$
\psi: L \rightarrow \operatorname{Com} K
$$

by setting

$$
x \psi=\Theta^{x} .
$$


From the definition of $\Phi^{x}$, if $x \leq y$, then $\Phi^{x} \leq \Phi^{y}$, and so $\theta^{x} \leq \boldsymbol{\theta}^{y}$. Thus $\psi$ is isotone.

By Lemmas 9 and 11, $\psi$ is surjective; if $\Theta^{2}$ on $C^{2}$ extends to $K$, then $\boldsymbol{\Theta}=\Phi^{x}$, where $x=\bigvee(\mathfrak{p} \varphi \mid \boldsymbol{\theta}(\mathfrak{p}) \leq \boldsymbol{\theta})$.

Finally, we claim that

$$
\Phi^{x} \leq \Phi^{y} \quad \text { implies that } x \leq y .
$$

This is clear if $x=0$. Otherwise, there is a prime interval $[u, v]$ in $C$ with $[u, v] \varphi=x$, and so $u \equiv v\left(\Phi^{x}\right)$. Therefore, $u \equiv v\left(\Phi^{y}\right)$, which implies that $x \leq y$ by the definition of $\Phi^{y}$. In other words,

$$
x \psi \leq y \psi \text { implies that } x \leq y .
$$

Consequently, $\psi$ is an isomorphism. This concludes the proof of the lemma, and of Theorem 8.

\section{The main result}

In this section, we state and prove the main result of this paper. The construction we are about to present is similar to, but more complicated than, the construction in Section 6, even if we ignore automorphisms.

Let $K$ be an m-complete lattice. Then the lattice of all m-complete congruence relations of $K$ is an m-algebraic lattice, in which, for any $a, b \in K$, the smallest $\mathfrak{m}$-complete congruence relation collapsing $a$ and $b$ is an $\mathfrak{m}$ compact element. In particular, if $K$ is bounded (that is, $K$ has a zero, 0 , and a unit, 1 ), then $l$ is m-compact.

Our main result is the converse statement.

THEOREM 13. Let $\mathfrak{m}$ be an uncountable regular cardinal. Let $L$ be an $\mathfrak{m}$-algebraic lattice with more than one element whose unit element 1 is $\mathfrak{m}$ compact, and let $G$ be a group. Then there exists a bounded m-complete lattice $K$ whose lattice of $\mathrm{m}$-complete congruence relations is isomorphic to $L$, and whose automorphism group is isomorphic to $G$.

Let $S_{\mathrm{m}}(L)$ denote the set of nonempty subsets $\mathrm{X}$ of $\operatorname{comp}_{\mathrm{m}}$ L with $|X|<$ $\mathrm{m}$. Let $X \in S_{\mathrm{m}}(L)$. Since $L$ has more than one element, it follows that $S_{\mathrm{m}}(L) \neq \varnothing$. We define a chain $C^{X}$ with unit $1^{X}$ and zero $j^{X}$ as follows.

Well-order $X$ by setting $X=\left\{x_{\gamma} \mid \gamma<\zeta^{X}\right\}$, where $\zeta^{X}<\mathfrak{m}$, and set

$$
C^{X}=(\omega \times X)+\mathfrak{C}_{1} .
$$


We define the coloring $\varphi^{X}$ of $C^{X}$,

$$
\varphi^{X}: \operatorname{Ip} C^{X} \rightarrow X
$$

by setting

$$
\left[\left\langle i, x_{\gamma}\right\rangle, u\right] \varphi^{X}=x_{\gamma}
$$

where

$$
u= \begin{cases}\left\langle i, x_{\gamma+1}\right\rangle ; & \text { if } \gamma+1<\zeta^{X} \\ \left\langle i+1, x_{0}\right\rangle ; & \text { if } \gamma+1=\zeta^{X} .\end{cases}
$$

Note that $\varphi^{X}$ is a repetitious coloring (see Section 5) of $C^{X}$. (Observe that the chain $X^{\dagger}$ of [8] can be obtained by adjoining a new zero element to $C^{X}$.)

Let us call a lattice (automorphism) rigid if it only has the trivial automorphism. We shall use the following result.

LEMMA 14. There are arbitrarily large sets of pairwise nonisomorphic rigid lattices of length 3.

Proof. Let $\mathfrak{G}=\langle V, E\rangle$ denote a symmetric graph (with no loops), where $V$ is the set of vertices and $E$ is the set of edges. $\mathfrak{G}$ is (automorphism) rigid if it only has the trivial automorphism. In A. Pultr and V. Trnkova [12, Chapter II.4], arbitrarily large sets of pairwise nonisomorphic rigid graphs are constructed.

Following $R$. Frucht [2], with any symmetric graph $\mathfrak{G}$ we associate a lattice $H(\mathfrak{B})$ of length 3 by setting

$$
H(\mathfrak{G})=V \dot{\cup} E \dot{\cup}\{0,1\} ;
$$

we partially order $H(\mathfrak{G})$ as follows: for $v \in V$ and $e \in E$, let $0<v<1$, $0<e<1$, and let $v<e$ if $v \in e$. Any automorphism of the lattice $H(\mathfrak{G})$ yields an automorphism of the graph $\mathfrak{G}$; thus the only automorphism of the lattice $H(\mathfrak{G})$ is the identity mapping. Similarly, $H\left(\mathfrak{G}_{0}\right) \cong H\left(\mathfrak{G}_{1}\right)$ implies that $\mathfrak{G}_{0} \cong \mathfrak{G}_{1}$. The lemma is thus proved.

Using Lemma 14, for each $X \in S_{\mathrm{m}}(L)$, we choose a rigid lattice $M_{X}$ of length 3 such that if $X, Y \in S_{\mathrm{m}}(L)$ and $X \neq Y$, then $M_{X}$ and $M_{Y}$ are not isomorphic. Let the lattice $X^{*}$ be constructed by adding the chain $C^{X}$ to the top of $M_{X}$, identifying the unit element of $M_{X}$ with $j^{X}$ (see Figure 2). Denote the zero element of $X^{*}$ by $0^{X}$. To put it into the context of Section 6, $X^{*}$ is obtained by inserting $M_{X}$ into the prime interval $\left[0^{X}, j^{X}\right]$ of the chain $X^{\dagger}$. 


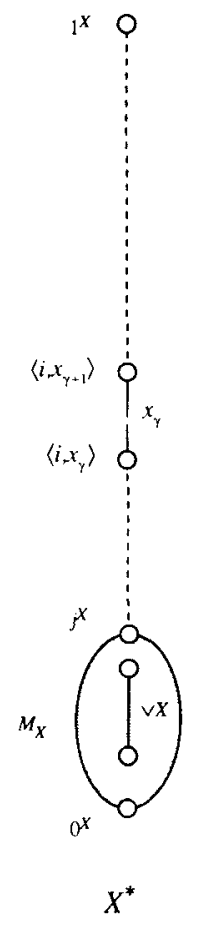

FIGURE 2

We shall construct two lattices $A_{0}$ and $A_{1}$. The lattice $A_{0}$ will contain the constructs that force the infinite joins to work properly. The lattice $A_{1}$ has only one role: for each color $x$, to have a prime interval of that color. Unfortunately, since we color with comp $\mathrm{m}_{\mathrm{m}} \mathrm{L}$, we cannot make $A_{1}$ into a chain.

We first construct the lattice $A_{0}$ :

$$
A_{0}=\bigcup\left(X^{*} \mid X \in S_{\mathrm{m}}(L)\right) \dot{\cup}\{0,1\},
$$

where we set $0<a<1$ whenever $a \in A_{0}-\{0,1\}$, and, if $a, b \in A_{0}-$ $\{0,1\}$, then $a<b$ if and only if $a, b \in X^{*}$ for some $X$ and $a<b$ in the lattice $X^{*}$ (see Figure 3).

To construct the lattice $A_{1}$, let us now fix an element of comp ${ }_{\mathrm{m}} \mathrm{L}$, say 1 . For each $x \in \operatorname{comp}_{\mathfrak{m}} L, x \neq 1$, choose a rigid lattice $N_{x}$ of length 3 such that if $x \neq y$, then $N_{x}$ is not isomorphic to $N_{y}$, and such that no $N_{x}$ is isomorphic to any $M_{X}$ whenever $x \in \operatorname{comp}_{\mathrm{m}} L, x \neq 1$ and $X \in S_{\mathrm{m}}(L)$. Finally, by the results of R. Frucht [1] and G. Sabidussi [13], we can represent the group $G$ as the automorphism group of a symmetric graph $\mathfrak{G}$. Define the lattice $N_{1}$ as $H(\mathfrak{G})$. Then $N_{1}$ is a lattice of length 3 whose automorphism 


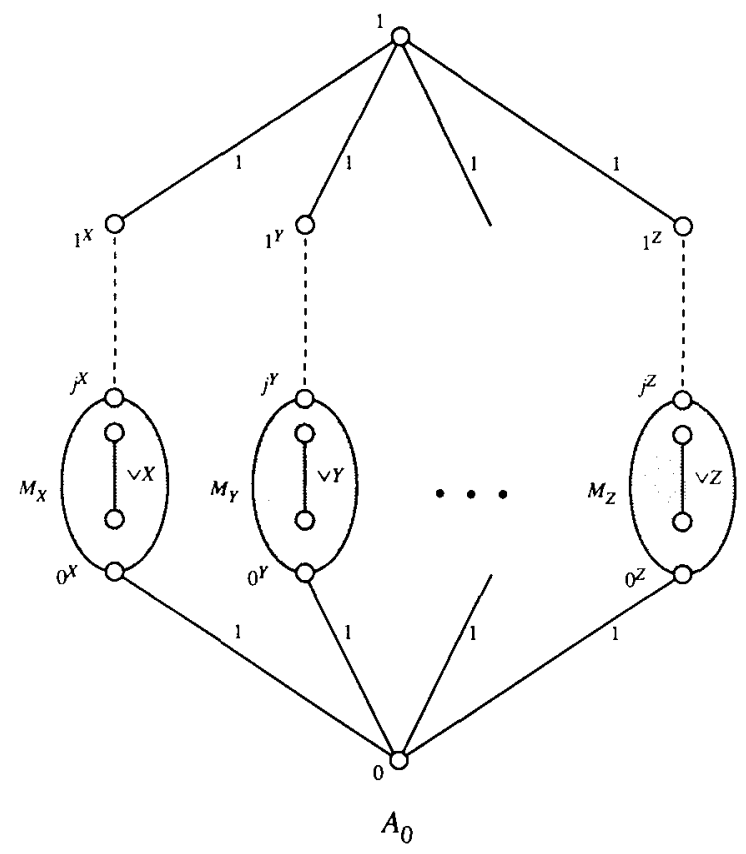

FIGURE 3

group is isomorphic to $G$. It is obvious that $N_{1}$ can also be taken so as not to be isomorphic to any other $N_{x}$ nor to any $M_{X}$ whenever $x \in \operatorname{comp}_{\mathrm{m}} L$ and $X \in S_{\mathrm{m}}(L)$. Let $u_{x}$ denote the zero of $N_{x}$ and let $v_{x}$ denote the unit of $N_{x}$.

We construct the lattice $A_{1}$ as follows:

$$
A_{1}=\bigcup\left(N_{x} \mid x \in \operatorname{comp}_{\mathrm{m}} L\right) \dot{U}\{0,1\} ;
$$

we partially order $A_{1}$ as follows: for $a \in A_{1}-\{0,1\}$, we set $0<a<1$; for $a, b \in A_{1}-\{0,1\}$, let $a<b$ if and only if there exists an $x \in \operatorname{comp}_{\mathrm{m}} L$ with $a, b \in N_{x}$ and $a<b$ in the lattice $N_{x}$ (see Figure 4).

Note that $A_{1}$ has length 5 and that all chains in $A_{0}$ are of cardinality less than $\mathrm{m}$. It then follows from Condition (2.3) that all m-complete congruence relations on $A_{0}$ and $A_{1}$ are complete. Since both $A_{0}$ and $A_{1}$ are strongly atomic, we get the following consequence of Lemma 9 .

LEMMA 15. For $i=0$ or 1 , the equality

$$
\mathrm{pi}_{\mathrm{m}} \boldsymbol{\theta}=\boldsymbol{\theta}
$$

holds for any m-complete congruence relation $\Theta$ on $A_{i}$. 


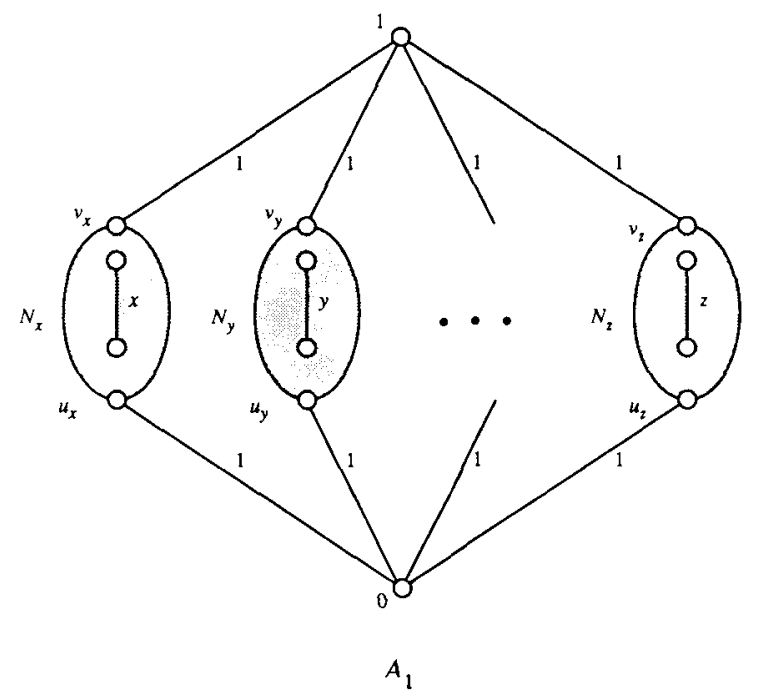

Figure 4

We color the lattices $A_{0}$ and $A_{1}$. Let

$$
P_{0}=\left(\operatorname{Ip} A_{0}\right) \cup\left\{\left[0^{X}, 1^{X}\right] \mid X \in S_{\mathrm{m}}(L)\right\},
$$

and set

$$
P_{1}=\operatorname{Ip} A_{1} \text {. }
$$

Define the coloring

$$
\varphi_{0}: P_{0} \rightarrow \operatorname{comp}_{\mathfrak{m}} L
$$

by setting, for $X \in S_{\mathfrak{m}}(L)$,

$$
\left[0^{X}, 1^{X}\right] \varphi_{0}=\bigvee X
$$

and setting, for $\mathfrak{p} \in \operatorname{Ip} A_{0}$ and $X \in S_{\mathfrak{m}}(L)$,

$$
\mathfrak{p} \varphi_{0}= \begin{cases}\mathfrak{p} \varphi^{X}, & \text { if } \mathfrak{p} \in \operatorname{Ip}\left[j^{X}, 1^{X}\right] \\ \vee X, & \text { if } \mathfrak{p} \in \operatorname{Ip} M_{X} ; \\ 1, & \text { if } \mathfrak{p}=\left[0,0^{X}\right] \text { or } \mathfrak{p}=\left[1^{X}, 1\right] .\end{cases}
$$

Note that $\varphi_{0}$ maps $P_{0}$ onto comp ${ }_{m} L$.

The coloring

$$
\varphi_{1}: P_{1} \rightarrow \operatorname{comp}_{\mathrm{m}} L
$$

is defined by setting

$$
\mathfrak{p} \varphi_{1}= \begin{cases}x, & \text { if } \mathfrak{p} \in \operatorname{Ip} N_{x} \\ 1, & \text { if } \mathfrak{p}=\left[0, u_{x}\right] \text { or } \mathfrak{p}=\left[v_{x}, 1\right] ;\end{cases}
$$


for $\mathfrak{p} \in \operatorname{Ip} A_{1}$ and $x \in \operatorname{comp}_{\mathfrak{m}} L$. Note that $\varphi_{1}$ maps $P_{1}$ onto comp $\mathrm{m}$.

For $i=0,1$, we observe that the following condition $(\Lambda)$ and its dual ( $V$ ) hold:

$(\bigwedge)$ if $[u, w] \in \operatorname{Ip} A_{i}$ and $y<w$, then there is a $v$ with $y \leq v \prec w$ and $[v, w] \varphi_{i}=[u, w] \varphi_{i}$.

For strongly atomic lattices, Condition $(V)$ is equivalent to the following condition:

if $u \prec v$ and $u \prec w$, then $[u, v] \varphi=[u, w] \varphi$.

In this condition, $\varphi$ is a coloring such that all prime intervals are in $P$. We call this condition ( $\mathrm{V}$ ) because the elements $u, v$, and $w$ form a $\mathrm{V}$.

The dual condition $(\Lambda)$ combines the dual of the above condition with a weak version of dual strong atomicity.

The coloring of the prime intervals in $A_{0}$ and $A_{1}$ are depicted in Figures 2-4 by labels to the right of the covering edges.

Lemma 16. An m-complete congruence relation $\boldsymbol{\theta}=\boldsymbol{\Theta}_{0} \times \boldsymbol{\Theta}_{1}$ on $A_{0} \times A_{1}$ has an extension to $A_{0} \times_{\varphi} A_{1}$ if and only if the following two conditions hold:

(1) If $\mathfrak{p}_{0} \in \operatorname{Ip} A_{0}, \mathfrak{p}_{1} \in \operatorname{Ip} A_{1}$, and $\mathfrak{p}_{0} \varphi_{0}=\mathfrak{p}_{1} \varphi_{1}$, then

$$
\boldsymbol{\Theta}\left(\mathfrak{p}_{0}\right) \leq \boldsymbol{\Theta}_{0} \quad \text { is equivalent to } \quad \boldsymbol{\Theta}\left(\mathfrak{p}_{1}\right) \leq \boldsymbol{\Theta}_{1}
$$

(2) If $P \subseteq \operatorname{Ip} A_{0}, q \in \operatorname{Ip} A_{0}$, and $q \varphi_{0} \leq \bigvee P \varphi_{0}$, then

$$
\boldsymbol{\Theta}(P) \leq \boldsymbol{\Theta}_{0} \text { implies that } \boldsymbol{\Theta}(\mathfrak{q}) \leq \boldsymbol{\Theta}_{0} .
$$

In that event, the extension is unique and $\mathrm{m}$-complete.

Proof. We use the Colored Product Extension Theorem; we have to show the equivalence of the conjunction of Conditions (16.1) and (16.2) with the conjunction of Conditions (7.1), (7.2), and the dual of (7.2).

Let Conditions (16.1) and (16.2) hold.

By Condition (16.1), Condition (7.1) holds for a prime interval $I_{0}$. Let $X \in S_{\mathrm{m}}(L)$; it is sufficient to consider an interval of the form $I_{0}=\left[0^{X}, 1^{X}\right]$. Choose an element $u$ in $M_{X}$ covering $0^{X}$, and a prime interval $I_{1}$ of $A_{1}$ with $I_{1} \varphi_{1}=\bigvee X$. Since

$$
\left[0^{X}, u\right] \varphi_{0}=\bigvee X=I_{0} \varphi_{0}=I_{1} \varphi_{1},
$$

we conclude by (16.1) that

$$
\Theta\left(\left[0^{X}, u\right]\right) \leq \Theta_{0} \quad \text { is equivalent to } \quad \Theta\left(I_{1}\right) \leq \Theta_{1} .
$$

But, setting $P=\left\{\left[0^{X}, u\right]\right\}$, we conclude by (16.2) and Lemma 15 that

$$
\boldsymbol{\theta}\left(\left[0^{X}, u\right]\right) \leq \Theta_{0} \quad \text { is equivalent to } \boldsymbol{\theta}\left(\left[0^{X}, 1^{X}\right]\right) \leq \boldsymbol{\Theta}_{0} .
$$


Thus $\boldsymbol{\Theta}\left(I_{0}\right) \leq \boldsymbol{\Theta}_{0}$ if and only if $\boldsymbol{\Theta}\left(I_{1}\right) \leq \boldsymbol{\Theta}_{1}$, concluding our verification of Condition (7.1).

We now establish Condition (7.2) and its dual. By Conditions $(\Lambda),(\vee)$, and (16.1), Condition (7.2) and its dual hold for a prime interval $I$. On the other hand, for $X \in S_{\mathrm{m}}(L)$, if $I=\left[0^{X}, 1^{X}\right]$ and $y<1^{X}$, then there is a $u \geq y$ with $j^{X} \leq u<1^{X}$. Therefore, $y \equiv 1^{X}\left(\Theta_{0}\right)$ implies that

$$
u \equiv 1^{X} \quad\left(\Theta_{0}\right) .
$$

Since $\varphi_{0}$ is repetitious, this congruence, Condition (16.2) with $P=\operatorname{Ip}\left[u, 1^{X}\right]$, and Lemma 15 imply that $0^{X} \equiv 1^{X}\left(\Theta_{0}\right)$, establishing (7.2).

As for the dual of (7.2), if $y>0^{X}$ with $y \equiv 0^{X}\left(\Theta_{0}\right)$, then there is in $M_{X}$ a cover $u$ of $0^{X}$ with $u \leq y$. Then, as above, taking $P=\left\{\left[0^{X}, u\right]\right\}$, we again get $0^{X} \equiv 1^{X}\left(\theta_{0}\right)$, establishing the dual of (7.2).

Now let Conditions (7.1), (7.2), and the dual of (7.2) hold. Condition (16.1) then follows immediately. Condition (7.1) implies that

(+) If $I, J \in P_{i}$ and $I \varphi_{i}=J \varphi_{i}$, then

$$
\boldsymbol{\theta}(I) \leq \boldsymbol{\theta}_{i} \text { is equivalent to } \boldsymbol{\theta}(J) \leq \boldsymbol{\theta}_{i} .
$$

Now we verify Condition (16.2). Since $q \varphi_{0}$ is m-compact, there is a nonempty subset $P^{\prime}$ of $P$ with $\left|P^{\prime} \varphi_{0}\right|<\mathfrak{m}$ and $\mathfrak{q} \varphi_{0} \leq \bigvee P^{\prime} \varphi_{0}$. Set $X=P^{\prime} \varphi_{0}$, $Y=X \cup\left\{\mathfrak{q} \varphi_{0}\right\}$; then $X, Y \in S_{\mathfrak{m}}(L)$. Since $\boldsymbol{\theta}(P) \leq \boldsymbol{\Theta}_{0}$, we get $\boldsymbol{\theta}\left(P^{\prime}\right) \leq \boldsymbol{\theta}_{0}$. By Conditions (7.1) and (+), and by Lemma 15 , we conclude that

$$
j^{X} \equiv 1^{X} \quad\left(\Theta_{0}\right) .
$$

Then, by (7.2) with $y=j^{X}$, we get that

$$
0^{X} \equiv 1^{X} \quad\left(\Theta_{0}\right) .
$$

Consequently, by (+),

$$
0^{Y} \equiv 1^{Y} \quad\left(\boldsymbol{\Theta}_{0}\right)
$$

Since $\mathfrak{q}_{0} \in Y=\left(\operatorname{Ip}\left[j^{Y}, 1^{Y}\right]\right) \varphi_{0}$, there is a $\mathfrak{p} \in \operatorname{Ip}\left[j^{Y}, 1^{Y}\right]$ with $\mathfrak{p} \varphi_{0}=\mathfrak{q} \varphi_{0}$. Thus, again by Condition $(+)$, we get that $q \in \Theta_{0}$, establishing (16.2) and concluding the proof of Lemma 16.

We now prove Theorem 13. Set $K=A_{0} \times{ }_{\varphi} A_{1}$. We first show that $\operatorname{Com}_{\mathrm{m}} K$ is isomorphic to $L$. We proceed as in the proof of Theorem 8 .

For $x \in L-\{0\}$ and $i=0,1$, define a congruence relation $\Phi_{i}^{x}$ on $A_{i}$ by setting, for $u \leq v$,

$$
u \equiv v \quad\left(\Phi_{i}^{x}\right) \quad \text { if } \quad \mathfrak{p} \varphi_{i} \leq x \quad \text { whenever } \mathfrak{p} \in \operatorname{Ip}[u, v] .
$$


By Lemma 2, it is easy to see that $\Phi_{i}^{x}$ is indeed a congruence relation and that it is $m$-complete. Note also that the pair $\Phi_{0}^{x}, \Phi_{1}^{x}$ satisfies Conditions (16.1) and (16.2). Thus the m-complete congruence relation $\Phi_{0}^{x} \times \Phi_{1}^{x}$ on $A_{0} \times A_{1}$ extends uniquely to an m-complete congruence relation $\Theta^{x}$ on $K$. Define

$$
\psi: L \rightarrow \operatorname{Com}_{\mathfrak{m}} K
$$

by setting

$$
x \psi=\theta^{x} .
$$

From the definition of $\Phi_{i}^{x}$, if $x \leq y$, then $\Phi_{i}^{x} \leq \Phi_{i}^{y}$, and so $\Theta^{x} \leq \Theta^{y}$. Thus $\psi$ is isotone.

It is easy to see that $\psi$ is surjective: if $\boldsymbol{\theta}_{0} \times \theta_{1}$ extends to $\theta$ on $K$, then set

$$
x=\bigvee\left(\mathfrak{p} \varphi_{0} \mid \boldsymbol{\theta}(\mathfrak{p}) \leq \boldsymbol{\theta}_{0}\right) .
$$

Then, by (16.2) and Lemma 15, $\Theta_{0}=\Phi_{0}^{x}$. Again, by (16.1) and Lemma 15, $\boldsymbol{\theta}_{1}=\Phi_{1}^{x}$. Thus $\boldsymbol{\theta}=\boldsymbol{\theta}^{x}$.

Finally, we claim that

$$
\boldsymbol{\theta}^{x} \leq \boldsymbol{\theta}^{y} \quad \text { implies that } x \leq y .
$$

If $x=0$, this implication is clear. Otherwise, there is a prime interval $[u, v]$ in $A_{0}$ with $[u, v] \varphi_{0}=x$, and so with $u \equiv v\left(\Phi_{0}^{x}\right)$. Then

$$
u \equiv v \quad\left(\Phi_{0}^{y}\right) .
$$

By the definition of $\Phi_{0}^{y}$, the fact that $[u, v]$ is prime and $[u, v] \varphi_{0}=x$, this congruence implies that $x \leq y$. Thus

$$
x \psi \leq y \psi \text { implies that } x \leq y,
$$

concluding the proof that $\psi$ is an isomorphism. Thus

$$
L \cong \operatorname{Com}_{\mathrm{m}} K \text {. }
$$

We now determine the group of automorphisms Aut $K$ of $K$. With each automorphism $\varrho$ of $N_{1}$, we associate an automorphism $\varrho$ of $K$ by setting, for $\left\langle u_{0}, u_{1}\right\rangle \in A_{0} \times A_{1}$,

$$
\left\langle u_{0}, u_{1}\right\rangle \bar{\varrho}=\left\{\begin{array}{ll}
\left\langle u_{0}, u_{1} \varrho\right\rangle, & \text { if } u_{1} \in N_{1} \\
\left\langle u_{0}, u_{1}\right\rangle, & \text { if } u_{1} \notin N_{1}
\end{array} ;\right.
$$

and

$$
m\left(I_{0}, I_{1}\right) \bar{\varrho}= \begin{cases}m\left(I_{0}, I_{1} \varrho\right), & \text { if } I_{1} \in \operatorname{Ip} N_{1} ; \\ m\left(I_{0}, I_{1}\right), & \text { if } I_{1} \notin \operatorname{Ip} N_{1} .\end{cases}
$$

It follows easily that the mapping $\alpha$ defined by $\varrho \alpha=\bar{\varrho}$ is an embedding of the group Aut $N_{1}$ into Aut $K$. 
Now let $\varrho^{\prime}$ be an automorphism of $K$. Since the only doubly irreducible elements of $K$ are the $m\left(I_{0}, I_{1}\right)$, it follows that $\varrho^{\prime}$ restricts to an automorphism of $A_{0} \times A_{1}$. The atoms of $A_{0} \times A_{1}$ are all of the form $\left\langle 0^{X}, 0\right\rangle$ with $X \in S_{\mathrm{m}}(L)$, or of the form $\left\langle 0, u_{x}\right\rangle$ with $x \in \operatorname{comp}_{\mathrm{m}} L$. Under $\varrho^{\prime}$, an atom must map to an atom. Now each $\left\langle 0^{X}, 0\right\rangle$ lies below a join-irreducible element $\left\langle 1^{X}, 0\right\rangle$ of infinite height of $A_{0} \times A_{1}$, while no $\left\langle 0, u_{x}\right\rangle$ lies below any join-irreducible element of infinite height. Consequently, for each $X \in S_{\mathrm{m}}(L)$, there is a $Y \in S_{\mathrm{m}}(L)$ with $\left\langle 0^{X}, 0\right\rangle \varrho^{\prime}=\left\langle 0^{Y}, 0\right\rangle$; and for each $x \in \operatorname{comp}_{\mathfrak{m}} L$, there is a $y \in \operatorname{comp}_{\mathfrak{m}} L$ with $\left\langle 0, u_{x}\right\rangle \varrho^{\prime}=\left\langle 0, u_{y}\right\rangle$.

Each $\left\langle j^{Z}, 0\right\rangle$ in $A_{0} \times A_{1}$ is of height 4 . Therefore,

$$
\left[\left\langle 0^{X}, 0\right\rangle,\left\langle j^{X}, 0\right\rangle\right] \varrho^{\prime}=\left[\left\langle 0^{Y}, 0\right\rangle,\left\langle j^{Y}, 0\right\rangle\right] .
$$

By their choice, no two distinct $M_{X}$ and $M_{Y}$ are isomorphic. Thus for each $X \in S_{\mathfrak{m}}(L)$,

$$
\left\langle 0^{X}, 0\right\rangle \varrho^{\prime}=\left\langle 0^{X}, 0\right\rangle \text { and }\left\langle j^{X}, 0\right\rangle \varrho^{\prime}=\left\langle j^{X}, 0\right\rangle .
$$

For every $X \in S_{\mathrm{m}}(L)$, the lattice $M_{X}$ has only one automorphism; we conclude that $\varrho^{\prime}$ is the identity on each interval $\left[\left\langle 0^{X}, 0\right\rangle,\left\langle j^{X}, 0\right\rangle\right]$. Finally, the intervals $\left[\left\langle j^{X}, 0\right\rangle,\langle 1,0\rangle\right]$ are well-ordered chains; thus we conclude, further, that $\varrho^{\prime}$ is the identity mapping on all of $A_{0} \times\{0\}$.

Similarly, $\varrho^{\prime}$ is the identity mapping on $\{0\} \times\left(A_{1}-N_{1}\right)$, and so there is a $\varrho \in$ Aut $N_{1}$ such that for all $u \in N_{1}$ we have $\langle 0, u\rangle \varrho^{\prime}=\langle 0, u \varrho\rangle$. It follows easily that $\varrho^{\prime}=\bar{\varrho}$. Thus $\alpha$ is an isomorphism of Aut $N_{1}$ with Aut $K$. Consequently,

$$
G \cong \text { Aut } K \text {. }
$$

This concludes the proof of the main result.

\section{Concluding remarks}

Observe that the main result of this paper, Theorem 13, contains the main result of [8] (Theorem 8 of Section 6). Indeed, if $K$ is a complete lattice and $\mathfrak{m}>|K|$, then $K$ is m-algebraic and the unit element of $K$ is m-compact, hence Theorem 13 applies and yields Theorem 8.

It is a very important restriction that $m$ be an uncountable regular cardinal. Indeed, if $\mathfrak{m}=\aleph_{0}$, then an $\mathrm{m}$-complete lattice $K$ becomes a lattice $K$. Similarly, the lattice of $m$-complete congruence relations of $K$ becomes the congruence lattice of $K$. Hence if $\mathfrak{m}=\aleph_{0}$, then $L$ must be distributive. 
If one is interested in the lattice of $m$-complete congruences only (and not in automorphism groups), then the proof of Theorem 13 can be simplified by taking $M_{X}=N_{x}=\mathfrak{C}_{2}$ whenever $X \in S_{\mathrm{m}}(L)$ and $x \in \operatorname{comp}_{\mathrm{m}} L$.

The lattice $K$ we constructed in Section 7 has many interesting properties. We now list some of them:

AdDendum to Theorem 13. The lattice $K$ in Theorem 13 can be chosen to have the following additional properties:

(1) $K$ is complete;

(2) every chain in $K$ is of cardinality less than $\mathfrak{m}$;

(3) $K$ is atomic and dually atomic;

(4) every $\mathfrak{m}$-complete congruence relation of $K$ is a complete congruence relation;

(5) every $\mathfrak{m}$-compact congruence relation of $K$ can be generated by a prime interval;

(6) Every m-complete congruence relation other than 1 leaves 0 and 1 isolated; that is, for every $\boldsymbol{\Theta} \neq \mathrm{l}$, we have $[0] \Theta=\{0\}$ and $[1] \Theta=$ $\{1\}$.

The Independence Theorem of Related Structures, due to G. Grätzer and W. A. Lampe, states that the three related structures of an infinitary algebra are independent; see Appendix 7 of G. Grätzer [4]. In G. Grätzer [6], a "lattice theoretic" proof was presented for the case of complete lattices. The proof presented in [6] works whenever the lattice to be represented as the congruence lattice of an algebra can be represented as the lattice of complete congruence relations of a complete lattice having property (6) of the Addendum. Thus we obtain:

InDePendence Theorem of Related STRUCtures. Let $\mathfrak{m}$ be an uncountable regular cardinal. Let $L_{c}$ and $L_{s}$ be $\mathfrak{m}$-algebraic lattices with more than one element, let the unit element of $L_{c}$ be m-compact, and let $G$ be a group. Then there exists an infinitary algebra $\mathfrak{A}$ of characteristic $\mathfrak{m}$ such that the congruence lattice of $\mathfrak{A}$ is isomorphic to $L_{c}$, the subalgebra lattice of $\mathfrak{A}$ is isomorphic to $L_{s}$, and the automorphism group of $\mathfrak{A}$ is isomorphic to $G$.

The idea of the proof in Section 6 is easy to visualize since we deal with the direct product of two chains. In fact, in [8], we left most of the proof to the reader. Is there an advantage to the more formal approach in Section 6? As the reader will recall, we colored the interval $\left[0^{C}, a^{C}\right]$ by 1 . In [8], this never comes up in the discussion. In the proof in Section 6, the condition $\left[0^{C}, a^{C}\right] \varphi=1$ enters the computation when we prove in Lemma 11 the dual 
of Condition (3.1). It is interesting to note that if the interval $\left[0^{C}, a^{C}\right]$ is colored by anything other than 1 , then the construction fails. The formal approach in Section 6 may clarify this and many other similar points to the reader.

We deal with very wide lattices in Section 7 since we do not know how to arrange all the chains $C^{X}, X \in S_{\mathrm{m}}(L)$, in one chain and still control the $\mathfrak{m}$-complete congruences. We first hoped that the techiques we developed in [8] will apply to two lattices which are not chains. A simple coloring of $\left(\mathfrak{C}_{2}\right)^{2}$ and $\mathfrak{C}_{3}$ show that this is not true. This forced us to develop Sections 3-5.

It is curious that even though the techniques developed in this paper do not apply to the congruence lattice characterization problem of finitary algebras (and to the finitary case of the Independence Theorem of Related Structures of G. Grätzer and W. A. Lampe), they have applications to finite lattices. For instance, we apply the One Point Extension Theorem and the Colored Product Extension Theorem to show that every finite distributive lattice $D$ can be represented as the congruence lattice of a finite planar lattice $L$, and this planar lattice has $O\left(n^{3}\right)$ elements, while older proofs (R. P. Dilworth, G. Grätzer, E. T. Schmidt, and J. Berman) produced lattices with $O\left(2^{2 n}\right)$ elements, where $n$ is the number of join-irreducible elements in $D$. A number of such results were announced in G. Grätzer and H. Lakser [11]; see also the papers of G. Grätzer and H. Lakser [9] and [10].

We conclude by mentioning the main open problem: Can every m-algebraic lattice be represented as the lattice of $m$-complete congruence relations of a suitable $\mathfrak{m}$-complete lattice?

\section{References}

[1] R. Frucht, 'Herstellung von Graphen mit vorgegebener abstrakter Gruppe', Compos. Math. 6 (1938), 239-250.

[2] R. Frucht, 'Lattices with a given group of automorphisms', Canad. J. Math. 2 (1950), 417-419.

[3] G. Grätzer, General Lattice Theory, Academic Press, New York, N. Y., 1978; Birkhäuser Verlag, Basel;Akademie Verlag, Berlin.

[4] G. Grätzer, Universal Algebra, Second Edition, Springer-Verlag, New York, Heidelberg, Berlin, 1979.

[5] G. Grätzer, 'On the the automorphism group and the complete congruence lattice of a complete lattice', Abstracts of papers presented to the Amer. Math. Soc. 88T-06-215.

[6] G. Grätzer, "A "lattice theoretic" proof of the independence of the automorphism group, the congruence lattice, and subalgebra lattice of an infinitary algebra', Abstracts of papers presented to the Amer. Math. Soc. 88T-08-254; Algebra Universalis (to appear). 
[7] G. Grätzer and H. Lakser, 'On the m-complete congruence lattice and the automorphism group of an m-complete lattice', Abstracts of papers presented to the Amer. Math. Soc. 88T-06-253.

[8] G. Grätzer and H. Lakser, 'On complete congruence lattices of complete lattices', Trans. Amer. Math. Soc. (to appear).

[9] G. Grätzer and H. Lakser, 'Congruence lattices of planar lattices', Abstracts of papers presented to the Amer. Math. Soc. 89T-06-29; Acta Math. Hungar. (to appear).

[10] G. Grätzer and H. Lakser, 'Homomorphisms of distributive lattices as restrictions of congruences. II. Restrictions of automorphisms', Abstracts of papers presented to the Amer. Math. Soc. 89T-06-92; Manuscript. University of Manitoba (1989).

[11] G. Grätzer and H. Lakser, 'Congruence lattices, automorphism groups of finite lattices and planarity', C. R. Math. Rep. Acad. Sci. Canada 11 (1989), 137-142.

[12] A. Pultr and V. Trnková, Combinatorial, algebraic and topological representations of groups, semigroups and categories, Academia, Prague, 1980.

[13] G. Sabidussi, 'Graphs with given infinite groups', Monatsh. Math. 64 (1960), 64-67.

[14] S.-K. Teo, 'Representing finite lattices as complete congruence lattices of complete lattices', Abstracts of papers presented to the Amer. Math. Soc. 88T-06-207; Ann. Univ. Sci. Budapest. Eötvös Sect. Math. (to appear).

\section{University of Manitoba,}

Winnipeg, Manitoba R3T 2N2,

Canada 